\title{
Efficient Adaptive Receivers for Joint Equalization and Interference Cancellation in Multiuser Space-Time Block-Coded Systems
}

\author{
Waleed M. Younis, Student Member, IEEE, Ali H. Sayed, Fellow, IEEE, and Naofal Al-Dhahir, Senior Member, IEEE
}

\begin{abstract}
This paper develops low-complexity adaptive receivers for space-time block-coded (STBC) transmissions over frequency-selective fading channels. The receivers are useful for equalization purposes for single user transmissions and for joint equalization and interference cancellation for multiuser transmissions. The receivers exploit the rich code structure of STBC codes in order to deliver recursive-least-squares (RLS) performance at least-mean-squares (LMS) complexity. Besides reduced complexity, the proposed adaptive receivers also lower system overhead requirements.
\end{abstract}

Index Terms-Adaptive receiver, equalization, interference cancellation, multiantenna systems, multiuser systems, space-time block-code.

\section{INTRODUCTION}

$\mathbf{I}$ NCREASING system capacity without requiring additional bandwidth is of major significance for spectrally-efficient high-rate wireless communication systems. Space-time blockcodes (STBCs) help increase reliability over wireless networks [1], and they can achieve full diversity gains with simple linear processing at the receiver.

It was shown in [2] that $K$ co-channel users, each equipped with $N$ antennas and transmitting uncorrelated signals, can be detected with $N$-order diversity gains if the receiver is equipped with $N(K-1)+1$ antennas. However, the structure of STBC can be exploited to reduce the number of receive antennas. It was shown in [3] that only $K$ receive antennas are needed to provide $N$-order diversity gains and suppress $K-1$ co-channel users.

A simple interference cancellation scheme for two co-channel users employing the Alamouti STBC scheme [4] was already developed in [3]. It was shown that by using two transmit antennas for each user and two receive antennas at the base station, it is possible to double the system capacity by applying only linear processing at the receiver. This scheme has

Manuscript received December 16, 2002; revised May 13, 2002. This work was supported in part by the National Science foundation under Grants ECS9820765 and CCR-0208573 and by a gift from AT\&T Shannon Laboratory. Parts of the results presented in this work were previously reported in [12]-[14]. The associate editor coordinating the review of this paper and approving it for publication was Dr. Helmut Bölcskei.

W. M. Younis and A. H. Sayed are with the Department of Electrical Engineering, University of California, Los Angeles, CA 90095 USA (e-mail: sayed@ee.ucla.edu).

N. Al-Dhahir is with the Department of Electrical Engineering, University of Texas at Dallas, Richardson, TX 75083 USA.

Digital Object Identifier 10.1109/TSP.2003.818209 been extended to the multiuser case in [5], where it was shown that the system capacity can be further increased by allowing more co-channel users. This is a useful observation, especially given that transmit diversity signaling using the Alamouti scheme [4] has been adopted in several wireless standards such as WCDMA and CDMA2000. Some of the attractive features of this diversity scheme are the following.

- It achieves full spatial diversity at full transmission rate for any (real or complex) signal constellation.

- It does not require channel state information (CSI) at the transmitter (i.e., it is open loop).

- Maximum likelihood decoding involves only linear processing at the receiver (due to the orthogonal code structure), thus keeping user terminals simple.

However, when implemented over frequency-selective channels, which is the focus of this paper, the Alamouti scheme should be implemented at a block not symbol level, and it should be combined with an effective equalization scheme to realize additional multipath diversity gains without sacrificing full spatial diversity. Several block level STBC structures for frequency selective fading channels, including time-reversal space-time block codes (TR-STBC) [6] and orthogonal frequency division multiplexing STBC (OFDM-STBC) [7], have been proposed in the literature. Another low-complexity scheme that achieves a similar goal is the single-carrier frequency-domain-equalized (SC FDE) STBC described in [8]. This scheme combines the advantages of the Alamouti scheme with those of SC FDE [9], namely, low complexity [due to use of the fast Fourier tarnsform (FFT)] and reduced sensitivity, compared with OFDM, to carrier frequency offsets and nonlinear distortion (due to reduced peak to average ratio).

Now, coherent joint interference cancellation, equalization, and decoding of SC FDE-STBC transmissions require channel state information (CSI) at the receiver, which can be estimated using training sequences embedded in each block. Then, the optimum equalizer/decoder settings can be computed from the estimated CSI. An alternative to this channel-estimate-based approach is adaptive equalization/decoding that does not require CSI estimation and helps reduce system overhead. It also provides a tracking mechanism for time-variant channels.

The purpose of this paper is to develop efficient low-complexity adaptive receivers for joint interference cancellation, equalization, and decoding in a multiuser environment with STBC transmissions. The operation of the receivers will be described for both training and tracking modes, and they will 
be shown to deliver RLS performance at LMS complexity. We will consider single-user, two-user, and multiuser STBC transmissions. In all cases, frequency-selective channels are assumed with two antennas per user for transmission and one antenna per user for reception.

The paper is organized as follows. In Section II, we describe the structure of the space-time block code and review a scheme for nonadaptive equalization for the single-user scenario and other schemes for joint interference cancellation and equalization with two or more co-channel users. Adaptive receivers are then developed in Section III for both training and tracking modes. Simulation results for the EDGE cellular ${ }^{1}$ environment are presented in Section IV, and the paper is concluded in Section $\mathrm{V}$.

\section{Space-Time Block Codes for BroAdBAnd Channels}

For STBCs to achieve multipath and spatial diversity gains on frequency-selective channels, the Alamouti scheme should be implemented at a block not symbol level. In this section, we describe the relevant encoding rule and the corresponding linear minimum-mean-square-error (MMSE) receivers for both cases of single-user and two-users transmissions.

\section{A. Single-User Transmissions}

Consider the scheme shown in Fig. 1, where a single user equipped with two antennas transmits data over a wireless channel, and the receiver has a single antenna. Data are transmitted from the antennas in blocks of length $N$, each according to the space-time coding scheme indicated in Fig. 2. Denote the $n$th symbol of the $k$ th transmitted block from antenna $i$ by $\mathrm{x}_{i}^{(k)}(n)$. At times $k=0,2,4, \cdots$, pairs of blocks $\mathrm{x}_{1}^{(k)}(n)$ and $\mathrm{x}_{2}^{(k)}(n)(0 \leq n \leq N-1)$ are generated by an information source according to the rule [8]:

$$
\begin{aligned}
& \mathbf{x}_{1}^{(k+1)}(n)=-\mathbf{x}_{2}^{*(k)}\left((-n)_{N}\right) \\
& \mathbf{x}_{2}^{(k+1)}(n)=\mathbf{x}_{1}^{*(k)}\left((-n)_{N}\right)
\end{aligned}
$$

where the date vector $\mathbf{x}$ has a covariance matrix equal to $\sigma_{x}^{2} \mathbf{I}_{N}$, and where $(\cdot)^{*}$ and $(\cdot)_{N}$ denote complex conjugation and modulo- $N$ operations, respectively. In addition, a cyclic prefix of length $\nu$ is added to each transmitted block to eliminate inter-block interference (IBI) and to make all channel matrices circulant. Here, $\nu$ denotes the longest channel memory between the transmit antennas and the receive antennas. With two

${ }^{1}$ EDGE stands for enhanced data rates for global evolution. transmit and one receive antenna, the received blocks $k$ and $k+1$, in the presence of additive white noise, are described by

$$
\mathbf{y}^{(j)}=\mathbf{H}_{1}^{(j)} \mathbf{x}_{1}^{(j)}+\mathbf{H}_{2}^{(j)} \mathbf{x}_{2}^{(j)}+\mathbf{n}^{(j)} \text { for } j=k, k+1
$$

where $\mathbf{n}^{(j)}$ is the noise vector with covariance matrix $\sigma_{n}^{2} \mathbf{I}_{N}$, and $\mathbf{H}_{1}^{(j)}$ and $\mathbf{H}_{2}^{(j)}$ are the circulant channel matrices from the first and second transmit antennas, respectively, over block $j$, to the receive antenna. Specifically, each $\mathbf{H}_{i}^{(j)}$ has the form shown at the bottom of the page, in terms of the impulse response sequence $\mathbf{h}_{i}^{(j)} \triangleq\left[h_{i}^{(j)}(0), h_{i}^{(j)}(1), \cdots, h_{i}^{(j)}(\nu)\right]$. Applying the DFT matrix $\mathbf{Q}$ to $\mathbf{y}^{(j)}$, we get a relation in terms of frequency-transformed variables:

$$
\mathbf{Y}^{(j)} \triangleq \mathbf{Q} \mathbf{y}^{(j)}=\Lambda_{1}^{(j)} \mathbf{X}_{1}^{(j)}+\Lambda_{2}^{(j)} \mathbf{X}_{2}^{(j)}+\mathbf{N}^{(j)}
$$

where $\mathbf{X}_{i}^{(j)} \triangleq \mathbf{Q} \mathbf{x}_{i}^{(j)}, \mathbf{N}^{(j)} \triangleq \mathbf{Q} \mathbf{n}^{(j)}$, and $\Lambda_{1}^{(j)}$ and $\Lambda_{2}^{(j)}$ are diagonal matrices given by $\Lambda_{i}^{(j)} \triangleq \mathbf{Q H}_{i}^{(j)} \mathbf{Q}^{*}$. Using the encoding rule (1) and properties of the DFT [10] and assuming the two channels are fixed over two consecutive blocks, we have that

$$
\begin{aligned}
& \mathbf{X}_{1}^{(k+1)}(m)=-\mathbf{X}_{2}^{*(k)}(m) \\
& \mathbf{X}_{2}^{(k+1)}(m)=\mathbf{X}_{1}^{*(k)}(m)
\end{aligned}
$$

for $m=0,1, \cdots, N-1$ and $k=0,2,4, \cdots$. Combining (3) and (4), we arrive at the linear relation

$$
\begin{aligned}
\mathbf{Y} & =\left(\begin{array}{c}
\mathbf{Y}^{(k)} \\
\overline{\mathbf{Y}}^{(k+1)}
\end{array}\right)=\left(\begin{array}{cc}
\boldsymbol{\Lambda}_{1} & \boldsymbol{\Lambda}_{2} \\
\Lambda_{2}^{*} & -\boldsymbol{\Lambda}_{1}^{*}
\end{array}\right)\left(\begin{array}{l}
\mathbf{X}_{1}^{(k)} \\
\mathbf{X}_{2}^{(k)}
\end{array}\right)+\left(\begin{array}{c}
\mathbf{N}^{(k)} \\
\overline{\mathbf{N}}^{(k+1)}
\end{array}\right) \\
& \triangleq \boldsymbol{\Lambda} \mathbf{X}+\mathbf{N}
\end{aligned}
$$

where $(\bar{\cdot})$ denotes complex conjugation of the entries of the vector. Relation (5) tells us how the transformed received vectors $\left\{\mathbf{Y}^{(k)}, \mathbf{Y}^{(k+1)}\right\}$ are related to the transformed transmitted blocks $\left\{\mathbf{X}_{1}^{(k)}, \mathbf{X}_{2}^{(k)}\right\}$ from the two antennas. It turns out that the matrix $\Lambda$ in (5) has a very useful structure, viz., $\Lambda^{*} \Lambda$ is diagonal. This fact can be exploited to recover the $\left\{\mathbf{X}_{1}^{(k)}, \mathbf{X}_{2}^{(k)}\right\}$ from the noisy observations $\left\{\mathbf{Y}^{(k)}, \mathbf{Y}^{(k+1)}\right\}$ via a simple linear receiver structure. The matrix structure of $\boldsymbol{\Lambda}$ in (5), with diagonal submatrices $\left\{\boldsymbol{\Lambda}_{1}, \boldsymbol{\Lambda}_{2}\right\}$, will appear frequently throughout the paper, and we will use the terminology Alamouti-like matrix to refer to it. Indeed, if we multiply both sides of (5) by $\Lambda^{*}$, we can decouple the signals $\mathbf{X}_{1}^{(k)}$ and $\mathbf{X}_{2}^{(k)}$ since this step gives

$$
\tilde{\mathbf{Y}} \triangleq \Lambda^{*} \mathbf{Y}=\left(\begin{array}{cc}
\Lambda^{o} & 0 \\
0 & \Lambda^{o}
\end{array}\right)\left(\begin{array}{l}
\mathbf{X}_{1}^{(k)} \\
\mathbf{X}_{2}^{(k)}
\end{array}\right)+\tilde{\mathbf{N}}
$$

$$
\mathbf{H}_{i}^{(j)}=\left(\begin{array}{cccccc}
h_{i}^{(j)}(0) & 0 & \cdots & h_{i}^{(j)}(\nu) & \cdots & h_{i}^{(j)}(1) \\
\vdots & \ddots & \ddots & \ddots & \ddots & \vdots \\
h_{i}^{(j)}(\nu-1) & \cdots & h_{i}^{(j)}(0) & 0 & \cdots & h_{i}^{(j)}(\nu) \\
h_{i}^{(j)}(\nu) & h_{i}^{(j)}(\nu-1) & \cdots & h_{i}^{(j)}(0) & 0 & \cdots \\
\vdots & \ddots & \ddots & \ddots & \ddots & \vdots \\
0 & \cdots & h_{i}^{(j)}(\nu) & h_{i}^{(j)}(\nu-1) & \cdots & h_{i}^{(j)}(0)
\end{array}\right)
$$


where $\boldsymbol{\Lambda}^{o}$ is an $N \times N$ diagonal matrix with $(i, i)$ element equal to $\left|\boldsymbol{\Lambda}_{1}(i, i)\right|^{2}+\left|\boldsymbol{\Lambda}_{2}(i, i)\right|^{2}$, which is also equal to the sum of the squared $i$ th DFT coefficients of first and second channel impulse responses. The filtered noise vector $\tilde{\mathbf{N}}$ has a diagonal covariance matrix equal to $\operatorname{diag}\left(\boldsymbol{\Lambda}^{o}, \boldsymbol{\Lambda}^{o}\right)$. The minimum mean square error (MMSE) estimator of $\mathbf{X}$ given $\mathbf{Y}$ is now seen to be [11]

$$
\hat{\mathbf{X}}=\tilde{\Lambda} \Lambda^{*} \mathbf{Y}=\left(\Lambda^{*} \Lambda+\frac{1}{\operatorname{SNR}} \mathbf{I}_{2 N}\right)^{-1} \Lambda^{*} \mathbf{Y}
$$

where $\tilde{\Lambda}$ is diagonal, and SNR is the signal-to-noise ratio at the receiver $\mathrm{SNR}=\sigma_{x}^{2} / \sigma_{n}^{2}$. The resulting MMSE frequencydomain equalizer is shown in Fig. 3 [12].

\section{B. Two-User Transmissions}

By using a second receive antenna, we can double the number of users [13]. Consider the block diagram shown in Fig. 4. With two receive antennas and two users (each equipped with two antennas), (5) generalizes to

$$
\begin{aligned}
\left(\begin{array}{c}
\mathbf{Y}_{1}^{(k)} \\
\overline{\mathbf{Y}}_{1}^{(k+1)} \\
\hline \mathbf{Y}_{2}^{(k)} \\
\overline{\mathbf{Y}}_{2}^{(k+1)}
\end{array}\right)= & \left(\begin{array}{cc|cc}
\boldsymbol{\Lambda}_{11_{1}} & \boldsymbol{\Lambda}_{11_{2}} & \boldsymbol{\Lambda}_{12_{1}} & \boldsymbol{\Lambda}_{12_{2}} \\
\boldsymbol{\Lambda}_{11_{2}}^{*} & -\boldsymbol{\Lambda}_{11_{1}}^{*} & \boldsymbol{\Lambda}_{12_{2}}^{*} & -\boldsymbol{\Lambda}_{12_{1}}^{*} \\
\hline \boldsymbol{\Lambda}_{21_{1}} & \boldsymbol{\Lambda}_{21_{2}} & \boldsymbol{\Lambda}_{22_{1}} & \boldsymbol{\Lambda}_{22_{2}} \\
\boldsymbol{\Lambda}_{21_{2}}^{*} & -\boldsymbol{\Lambda}_{21_{1}}^{*} & \boldsymbol{\Lambda}_{22_{2}}^{*} & -\boldsymbol{\Lambda}_{22_{1}}^{*}
\end{array}\right) \\
& \times\left(\begin{array}{c}
\mathbf{X}_{1_{1}}^{(k)} \\
\mathbf{X}_{1_{2}}^{(k)} \\
\hline \mathbf{X}_{2_{1}}^{(k)} \\
\mathbf{X}_{2_{2}}^{(k)}
\end{array}\right)+\left(\begin{array}{c}
\mathbf{N}_{1}^{(k)} \\
\overline{\mathbf{N}}_{1}^{(k+1)} \\
\hline \mathbf{N}_{2}^{(k)} \\
\overline{\mathbf{N}}_{2}^{(k+1)}
\end{array}\right)
\end{aligned}
$$

where $\mathbf{Y}_{2}^{(k)}$ and $\overline{\mathbf{Y}}_{2}^{(k+1)}$ are the received signals at the second receive antenna at blocks $k$ and $k+1$, respectively, and $\mathbf{X}_{2_{1}}^{(k)}$ and $\mathbf{X}_{2_{2}}^{(k)}$ are the $k^{t h}$ transmitted blocks from the first and second antennas of the second user, respectively. In more compact form, (8) can be written as

$$
\left(\begin{array}{l|l}
\mathbf{Y}_{1} \\
\hline \mathbf{Y}_{2}
\end{array}\right)=\left(\begin{array}{ll}
\boldsymbol{\Lambda}_{11} & \boldsymbol{\Lambda}_{12} \\
\hline \Lambda_{21} & \boldsymbol{\Lambda}_{22}
\end{array}\right)\left(\begin{array}{l}
\mathbf{X}_{1} \\
\hline \mathbf{X}_{2}
\end{array}\right)+\left(\begin{array}{l}
\mathbf{N}_{1} \\
\hline \mathbf{N}_{2}
\end{array}\right)
$$

where $\mathbf{Y}_{i}$ is the processed signal from the $i$ th receive antenna, whereas $\mathbf{N}_{i}$ is the corresponding noise vector. Moreover, $\mathbf{X}_{i}$ consists of the two subvectors representing the size- $N$ DFTs of the information blocks transmitted from the $i$ th user's first and second transmit antennas at time $k$, i.e.,

$$
\mathbf{Y}_{i}=\left(\begin{array}{c}
\mathbf{Y}_{i}^{(k)} \\
\overline{\mathbf{Y}}_{i}^{(k+1)}
\end{array}\right), \quad \mathbf{X}_{i}=\left(\begin{array}{c}
\mathbf{X}_{i_{1}}^{(k)} \\
\mathbf{X}_{i_{2}}^{(k)}
\end{array}\right), \quad \mathbf{N}_{i}=\left(\begin{array}{c}
\mathbf{N}_{i_{1}}^{(k)} \\
\overline{\mathbf{N}}_{i_{2}}^{(k)}
\end{array}\right)
$$

and each $\boldsymbol{\Lambda}_{i j}$ is the Alamouti-like overall frequency domain channel matrix from the $i$ th user transmit antennas to the $j$ th receive antenna. The two users can be decoupled as follows:

$$
\begin{aligned}
\left(\begin{array}{l}
\mathbf{Z}_{1} \\
\mathbf{Z}_{2}
\end{array}\right) & \triangleq\left(\begin{array}{cc}
\mathbf{I}_{2 N} & -\boldsymbol{\Lambda}_{12} \boldsymbol{\Lambda}_{22}^{-1} \\
-\boldsymbol{\Lambda}_{21} \boldsymbol{\Lambda}_{11}^{-1} & \mathbf{I}_{2 N}
\end{array}\right)\left(\begin{array}{l}
\mathbf{Y}_{1} \\
\mathbf{Y}_{2}
\end{array}\right) \\
& =\left(\begin{array}{cc}
\boldsymbol{\Sigma} & 0 \\
0 & \boldsymbol{\Delta}
\end{array}\right)\left(\begin{array}{l}
\mathbf{X}_{1} \\
\mathbf{X}_{2}
\end{array}\right)+\left(\begin{array}{l}
\tilde{\mathbf{N}}_{1} \\
\mathbf{N}_{2}
\end{array}\right)
\end{aligned}
$$

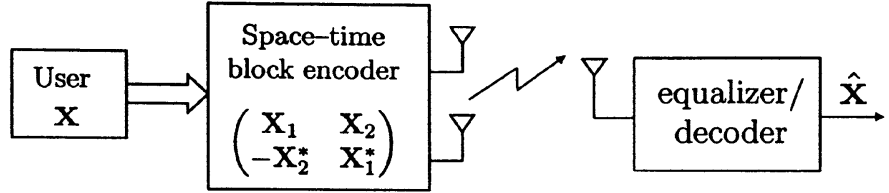

Fig. 1. Single-user system with two-transmit one-receive antenna.

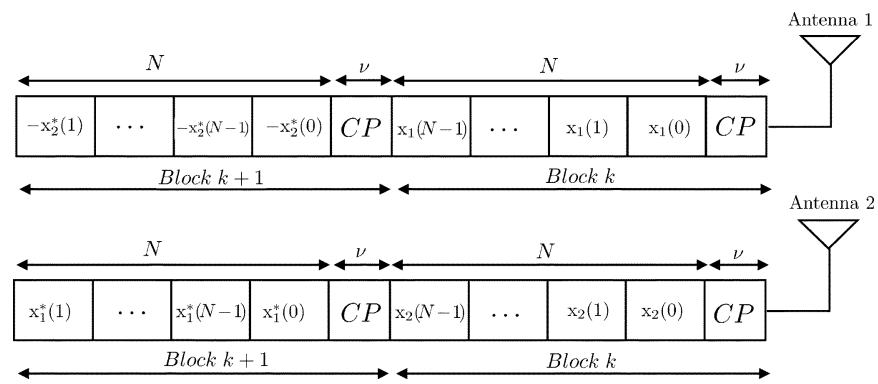

Fig. 2. Block format for SC FDE-STBC transmission scheme.

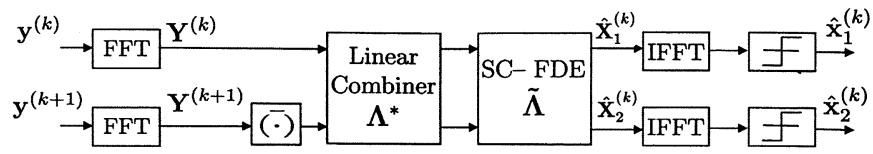

Fig. 3. Receiver structure for a two-transmit one-receive antenna system.

where $\boldsymbol{\Sigma}=\boldsymbol{\Lambda}_{11}-\boldsymbol{\Lambda}_{12} \boldsymbol{\Lambda}_{22}^{-1} \Lambda_{21}$, and $\boldsymbol{\Delta}=\boldsymbol{\Lambda}_{22}-\boldsymbol{\Lambda}_{21} \boldsymbol{\Lambda}_{11}^{-1} \boldsymbol{\Lambda}_{12}$. It can be verified that both $\Sigma$ and $\Delta$ have an Alamouti-like structure, i.e.,

$$
\Sigma=\left(\begin{array}{cc}
\Sigma_{1} & \Sigma_{2} \\
\Sigma_{2}^{*} & -\Sigma_{1}^{*}
\end{array}\right) \text { and } \Delta=\left(\begin{array}{cc}
\Delta_{1} & \Delta_{2} \\
\Delta_{2}^{*} & -\Delta_{1}^{*}
\end{array}\right)
$$

where $\left\{\Sigma_{1}, \Sigma_{2}, \Delta_{1}, \Delta_{2}\right\}$ are all diagonal. Once the two users have been decoupled, the equalization procedure described by (7) can be applied to each user $\left\{\mathbf{Z}_{1}, \mathbf{Z}_{2}\right\}$ in order to recover the original data $\left\{\mathbf{X}_{1}, \mathbf{X}_{2}\right\}$ (see [13]).

\section{Multiuser Case}

With $M$ users (each equipped with two antennas), we can use $M$ receive antennas to decouple all users and, hence, increase the system capacity. Equation (5) generalizes to

$$
\begin{aligned}
& \underbrace{\left(\begin{array}{c}
\mathbf{Y}_{1} \\
\mathbf{Y}_{2} \\
\vdots \\
\mathbf{Y}_{M}
\end{array}\right)}_{2 N M \times 1}=\underbrace{\left(\begin{array}{cccc}
\boldsymbol{\Lambda}_{11} & \boldsymbol{\Lambda}_{12} & \ldots & \boldsymbol{\Lambda}_{1 M} \\
\boldsymbol{\Lambda}_{21} & \boldsymbol{\Lambda}_{22} & \ldots & \boldsymbol{\Lambda}_{2 M} \\
\vdots & \vdots & \ddots & \vdots \\
\boldsymbol{\Lambda}_{M 1} & \boldsymbol{\Lambda}_{M 2} & \ldots & \boldsymbol{\Lambda}_{M M}
\end{array}\right)}_{2 N M \times 2 N M} \underbrace{\left(\begin{array}{c}
\mathbf{X}_{1} \\
\mathbf{X}_{2} \\
\vdots \\
\mathbf{X}_{M}
\end{array}\right)}_{2 N M \times 1} \\
& +\underbrace{\left(\begin{array}{c}
\mathbf{N}_{1} \\
\mathbf{N}_{2} \\
\vdots \\
\mathbf{N}_{M}
\end{array}\right)}_{2 N M \times 1}
\end{aligned}
$$

where $\mathbf{Y}_{i}, \mathbf{X}_{i}$, and $\mathbf{N}_{i}$ are given by (10), and each $\boldsymbol{\Lambda}_{i j}$ is the Alamouti-like frequency domain channel matrix from the $i$ th user transmit antennas to the $j$ th receive antenna. We can iteratively recover the symbols of each user by successive Schur complementation, starting from the $M$ th user, proceeding to the 


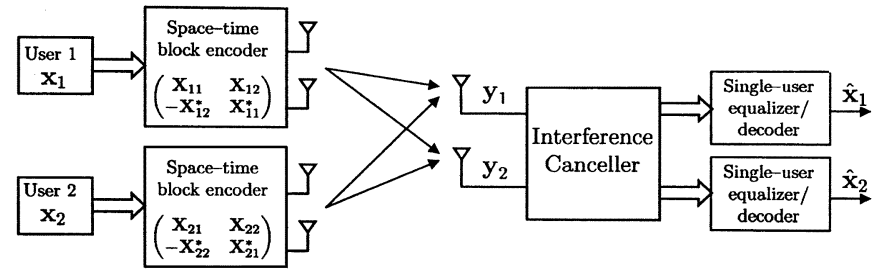

Fig. 4. Two-user system with two-transmit antennas per user and two-receive antennas.

$(M-1)$ th user, and down to the first user. For example, to recover the symbols of the $M$ th user, we partition the channel matrix in (12) as

$$
\left(\begin{array}{ccc|c}
\boldsymbol{\Lambda}_{11} & \boldsymbol{\Lambda}_{12} & \ldots & \boldsymbol{\Lambda}_{1 M} \\
\boldsymbol{\Lambda}_{21} & \boldsymbol{\Lambda}_{22} & \ldots & \boldsymbol{\Lambda}_{2 M} \\
\vdots & \vdots & \ddots & \vdots \\
\hline \boldsymbol{\Lambda}_{M 1} & \boldsymbol{\Lambda}_{M 2} & \ldots & \boldsymbol{\Lambda}_{M M}
\end{array}\right)=\left(\begin{array}{c|c}
\mathbf{A} & \mathbf{B} \\
\hline \mathbf{C} & \mathbf{D}
\end{array}\right)
$$

with $\mathbf{A}, \mathbf{B}, \mathbf{C}$, and $\mathbf{D}$ denoting the $2 N(M-1) \times 2 N(M-1)$ upper left, $2 N(M-1) \times 2 N$ upper right, $2 N \times 2 N(M-1)$ lower left, and $2 N \times 2 N$ lower right matrices, respectively. A linear interference canceller similar to the one designed for the two-user case in (11) can then be used to suppress the interference from the first $M-1$ users on the $M$ th user as follows:

$$
\begin{aligned}
\left(\begin{array}{c}
\mathbf{Z}_{1: M-1} \\
\hline \mathbf{Z}_{M}
\end{array}\right) \triangleq\left(\begin{array}{c|c} 
& \\
\mathbf{I}_{2 N \times(M-1)} & -\mathbf{B D}^{-1} \\
& \\
\hline-\mathbf{C A}^{-1} & \mathbf{I}_{2 N}
\end{array}\right)\left(\begin{array}{c}
\mathbf{Y}_{1} \\
\mathbf{Y}_{2} \\
\vdots \\
\hline \mathbf{Y}_{M}
\end{array}\right) \\
=\left(\begin{array}{c|c}
\mathbf{\Sigma}_{1: M-1} & \mathbf{0} \\
& \\
\hline \mathbf{0} & \mathbf{\Delta}_{M}
\end{array}\right)\left(\begin{array}{c}
\mathbf{X}_{1} \\
\mathbf{X}_{2} \\
\vdots \\
\hline \mathbf{X}_{M}
\end{array}\right)+\left(\begin{array}{c}
\tilde{\mathbf{N}}_{1} \\
\tilde{\mathbf{N}}_{2} \\
\vdots \\
\hline \tilde{\mathbf{N}}_{M}
\end{array}\right)
\end{aligned}
$$

where $\Sigma_{1: M-1}=\mathbf{A}-\mathbf{B D}^{-1} \mathbf{C}$, and $\boldsymbol{\Delta}_{M}=\mathbf{D}-\mathbf{C A}^{-1} \mathbf{B}$. It could be easily verified that $\boldsymbol{\Delta}_{M}$ has an Alamouti-like structure and that $\boldsymbol{\Sigma}_{1: M-1}$ is an $2 N(M-1) \times 2 N(M-1)$ block matrix with each $2 N \times 2 N$ block being an Alamouti-like matrix. This result follows from the following properties of Alamouti-like matrices:

- The sum or difference of two Alamouti-like matrices is an Alamouti-like matrix.

- The inverse of an Alamouti-like matrix is Alamouti-like.

- The inverse of a block matrix with Alamouti-like subblocks is a block matrix with Alamouti-like subblocks.

The next step is to apply the single-user SC MMSE-FDE of (7) to $\mathbf{Z}_{M}$ in order to get an estimate for the DFT of the symbols of the $M$ th user $\hat{\mathbf{X}}_{M}$. Then, we apply IDFT to $\hat{\mathbf{X}}_{M}$ to get an estimate for the corresponding symbols $\hat{\mathbf{x}}_{M}$.

The symbols of the $(M-1)$ th user can be recovered by repeating the procedure on the reduced system:

$$
\underbrace{\left(\mathbf{Z}_{1: M-1}\right)}_{2 N(M-1) \times 1}=\underbrace{\left(\boldsymbol{\Sigma}_{1: M-1}\right)}_{2 N(M-1) \times 2 N(M-1)} \underbrace{\left(\begin{array}{c}
\mathbf{X}_{1} \\
\mathbf{X}_{2} \\
\vdots \\
\mathbf{X}_{M-1}
\end{array}\right)}_{2 N(M-1) \times 1}+\underbrace{\left(\begin{array}{c}
\tilde{\mathbf{N}}_{1} \\
\tilde{\mathbf{N}}_{2} \\
\vdots \\
\tilde{\mathbf{N}}_{M-1}
\end{array}\right)}_{2 N(M-1) \times 1}
$$

Again, we partition $\boldsymbol{\Sigma}_{1: M-1}$ in a way similar to (13) with $\mathbf{A}, \mathbf{B}$, $\mathbf{C}$, and $\mathbf{D}$ now being the $2 N(M-2) \times 2 N(M-2)$ upper left, $2 N(M-2) \times 2 N$ upper right, $2 N \times 2 N(M-2)$ lower left, and $2 N \times 2 N$ lower right matrices, respectively. We then apply an interference canceller scheme similar to (14), followed by a single-user SC MMSE-FDE to get an estimate for the symbols of the $(M-1)$ th user $\hat{\mathbf{x}}_{M-1}$. We then proceed until we recover the symbols of all $M$ users. The block diagram of the multiuser receiver is shown in Fig. 5 [14].

\section{ADAPTIVE SCHEME}

The joint interference cancellation and equalization techniques described in the previous sections require the channels to be known at the receiver in order to know the $\left\{\boldsymbol{\Lambda}_{i j}\right\}$. Usually, channel estimation is done by adding a training sequence to each transmitted block and using it to estimate the channel, which tends to increase the system overhead. Reduction of the system overhead requires using longer blocks, which may not be viable for channels with fast variations. These observations motivate us to develop adaptive receivers for joint interference cancellation and equalization purposes. Our interest is to develop adaptive structures with fast tracking/convergence abilities (like recursive-least-squares structures) and then to show how the special structure of the space-time block code can be exploited to obtain RLS performance at LMS complexity. One of the advantages of adaptive receivers is that they eliminate the need for adding a training sequence to each data block. They use a few training blocks during initialization, and then they can track channel variations in a decision-directed mode. In this way, the system overhead can be reduced. We start with the single-user transmissions case.

\section{A. Single-User Transmissions}

From Fig. 3, we note that the MMSE equalizer consists of a linear combiner $\Lambda^{*}$ followed by scaling by a diagonal matrix. We denote the combined matrix by $\mathbf{A}=\tilde{\mathbf{\Lambda}} \mathbf{\Lambda}^{*}$, which is seen to have the structure

$$
\mathbf{A}=\left(\begin{array}{cc}
\mathbf{A}_{1} & \mathbf{A}_{2} \\
\mathbf{A}_{2}^{*} & -\mathbf{A}_{1}^{*}
\end{array}\right)
$$

where $\mathbf{A}_{1}$ and $\mathbf{A}_{2}$ are diagonal matrices given by

$$
\begin{aligned}
& \mathbf{A}_{1}=\operatorname{diag}\left\{\frac{1}{\Lambda^{o}(i, i)+\frac{1}{\mathrm{SNR}}}\right\}_{i=0}^{N-1} \cdot \boldsymbol{\Lambda}_{1}^{*} \\
& \mathbf{A}_{2}=\operatorname{diag}\left\{\frac{1}{\boldsymbol{\Lambda}^{o}(i, i)+\frac{1}{\mathrm{SNR}}}\right\}_{i=0}^{N-1} \cdot \boldsymbol{\Lambda}_{2} .
\end{aligned}
$$




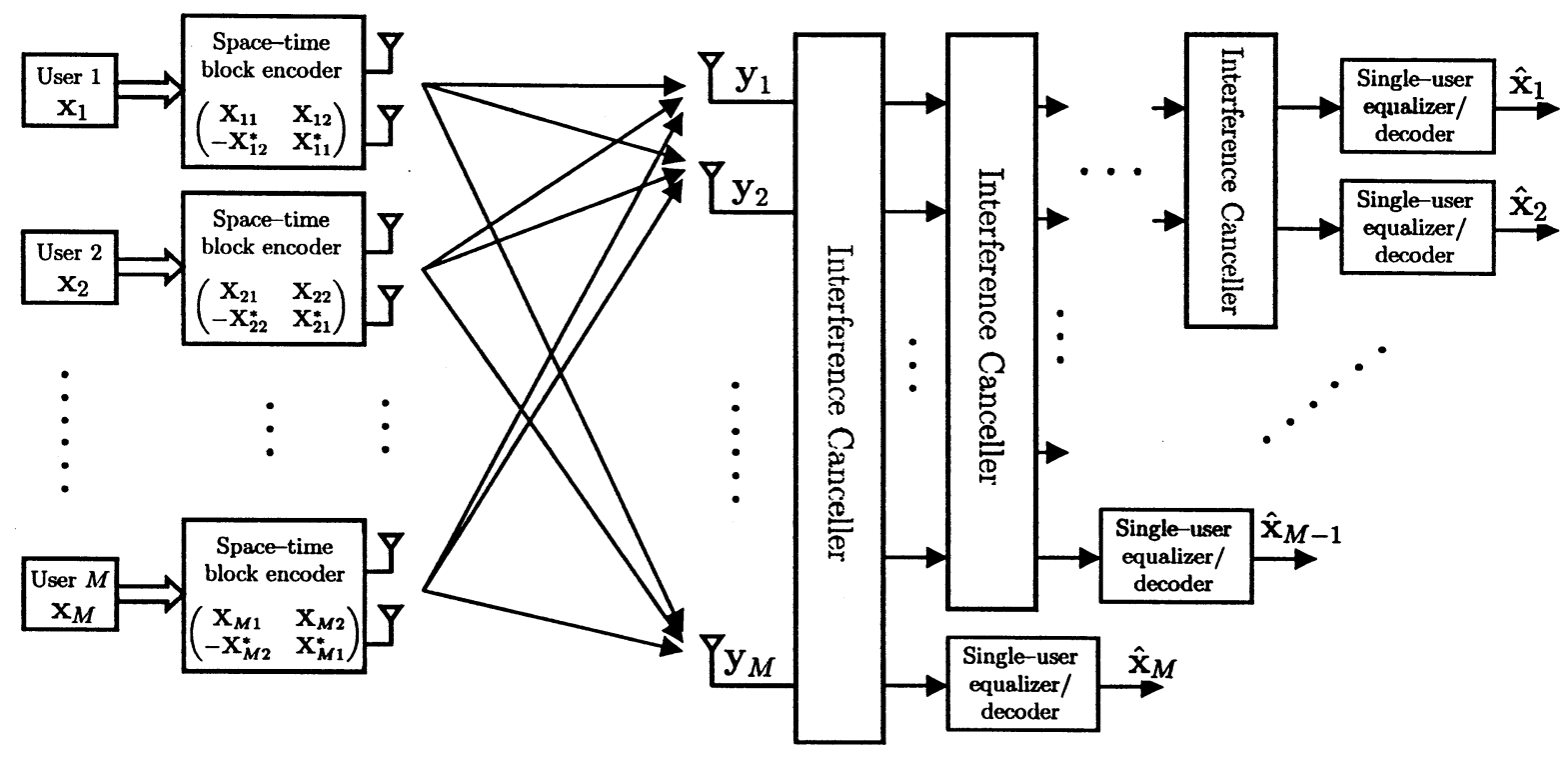

Fig. 5. Receiver structure for multiuser STBC transmissions.

Therefore, from Fig. 3, we have that

$$
\left(\begin{array}{c}
\hat{\mathbf{X}}_{1}^{(k)} \\
\hat{\mathbf{X}}_{2}^{(k)}
\end{array}\right)=\left(\begin{array}{cc}
\mathbf{A}_{1} & \mathbf{A}_{2} \\
\mathbf{A}_{2}^{*} & -\mathbf{A}_{1}^{*}
\end{array}\right) \mathbf{Y}
$$

which can be rewritten alternatively as

$$
\begin{aligned}
\left(\begin{array}{c}
\hat{\mathbf{X}}_{1}^{(k)} \\
\hat{\mathbf{X}}_{2}^{(k)}
\end{array}\right) & =\left(\begin{array}{cc}
\operatorname{diag}\left(\mathbf{Y}^{(k)}\right) & \operatorname{diag}\left(\overline{\mathbf{Y}}^{(k+1)}\right) \\
-\operatorname{diag}\left(\mathbf{Y}^{(k+1)}\right) & \operatorname{diag}\left(\overline{\mathbf{Y}}^{(k)}\right)
\end{array}\right)\left(\begin{array}{c}
\mathbf{W}_{1} \\
\mathbf{W}_{2}
\end{array}\right) \\
& \triangleq \mathbf{U}_{k} \mathcal{W}
\end{aligned}
$$

where $\mathbf{W}_{1}$ and $\mathbf{W}_{2}$ are the vectors containing the diagonal elements of $\mathbf{A}_{1}$ and $\mathbf{A}_{2}$, respectively. Moreover, $\mathcal{W}$ is a $2 N \times 1$ vector containing the elements of $\left\{\mathbf{W}_{1}, \mathbf{W}_{2}\right\}$, and $\mathbf{U}_{k}$ is an Alamouti-like matrix of size $2 N \times 2 N$ containing the received symbols from blocks $k$ and $k+1$. Equation (16) reveals the special structure of the STBC problem. In the nonadaptive scenario, the coefficients of $\mathcal{W}$ are calculated from a channel estimate at every block. Equation (16), on the other hand, suggests that $\mathcal{W}$ can be computed adaptively by using a block version of the RLS algorithm. Specifically, the equalizer coefficients are updated every two blocks, according to the following recursion:

$$
\mathcal{W}_{k+2}=\mathcal{W}_{k}+\mathcal{P}_{k+2} \mathbf{U}_{k+2}^{*}\left[\mathbf{D}_{k+2}-\mathbf{U}_{k+2} \mathcal{W}_{k}\right]
$$

where

$$
\begin{aligned}
\mathcal{P}_{k+2}= & \lambda^{-1}\left[\mathcal{P}_{k}-\lambda^{-1} \mathcal{P}_{k} \mathbf{U}_{k+2}\right. \\
& \left.\times\left(\mathbf{I}_{2 N}+\lambda^{-1} \mathbf{U}_{k+2} \mathcal{P}_{k} \mathbf{U}_{k+2}^{*}\right)^{-1} \mathbf{U}_{k+2}^{*} \mathcal{P}_{k}\right]
\end{aligned}
$$

where $\lambda$ is a forgetting factor that is usually close to 1 . The initial conditions are $\mathcal{W}_{0}=0$ and $\mathcal{P}=\delta \mathbf{I}_{2 N}, \delta$ is a large number, and
$\mathbf{I}_{2 N}$ is the $2 N \times 2 N$ identity matrix. Moreover, $\mathbf{D}_{k+2}$ is the desired response vector given by

$$
\mathbf{D}_{k+2}= \begin{cases}\left(\begin{array}{l}
\mathbf{X}_{1}^{(k+2)} \\
\overline{\mathbf{X}}_{2}^{(k+2)}
\end{array}\right), & \text { for training } \\
\left(\begin{array}{c}
\check{X}_{1}^{(k+2)} \\
\bar{X}_{2}^{(k+2)}
\end{array}\right), & \text { for decision-directed tracking. }\end{cases}
$$

It might seem that the computational complexity of the algorithm is high since matrix inversion is needed in (18). However, due to the special structure of the space-time block-code, no matrix inversion is needed and the complexity of the algorithm can be reduced to that of an LMS implementation. In this way, we are able to obtain RLS performance at LMS cost. The reasoning is as follows.

It follows by induction that $\mathcal{P}_{k+2}$ has a diagonal structure of the form:

$$
\mathcal{P}_{k+2}=\left(\begin{array}{cc}
\mathbf{P}_{k+2} & \mathbf{0} \\
\mathbf{0} & \mathbf{P}_{k+2}
\end{array}\right)
$$

where $\mathbf{P}_{k+2}$ is diagonal as well. This statement holds at time $k+2=0$ since, by assumption, $\mathcal{P}_{0}=\delta \mathbf{I}_{2 N}$ (so that $\left.\mathbf{P}_{0}=\delta \mathbf{I}_{N}\right)$. Now, assume the statement holds at time $k$. Then, it is easy to see that

$$
\left(\mathbf{I}_{2 N}+\lambda^{-1} \mathbf{U}_{k+2} \mathcal{P}_{k} \mathbf{U}_{k+2}^{*}\right)^{-1}=\left(\begin{array}{cc}
\boldsymbol{\Theta}_{k+2} & \mathbf{0} \\
\mathbf{0} & \boldsymbol{\Theta}_{k+2}
\end{array}\right)
$$

where $\boldsymbol{\Theta}_{k+2}$ is diagonal and given by

$$
\begin{aligned}
\boldsymbol{\Theta}_{k+2}= & {\left[\mathbf{I}_{N}+\lambda^{-1}\left(\operatorname{diag}\left(\mathbf{Y}^{(k)}\right) \mathbf{P}_{k} \operatorname{diag}\left(\overline{\mathbf{Y}}^{(k)}\right)\right.\right.} \\
& \left.\left.+\operatorname{diag}\left(\mathbf{Y}^{(k+1)}\right) \mathbf{P}_{k} \operatorname{diag}\left(\overline{\mathbf{Y}}^{(k+1)}\right)\right)\right]^{-1} .
\end{aligned}
$$

Since diagonal matrices commute, (20) simplifies to

$$
\boldsymbol{\Theta}_{k+2}=\left(\mathbf{I}_{N}+\lambda^{-1} \mathbf{P}_{k} \operatorname{diag}\left(\left|\mathbf{Y}^{(k)}\right|^{2}+\left|\mathbf{Y}^{(k+1)}\right|^{2}\right)\right)^{-1}
$$


TABLE I

ADAPTATION ALGORITHM FOR SINGLE-USER TRANSMISSIONS

Define

$$
\mathcal{P}_{k} \triangleq\left(\begin{array}{cc}
\mathbf{P}_{k} & \mathbf{0} \\
\mathbf{0} & \mathbf{P}_{k}
\end{array}\right)
$$

where $\mathbf{P}_{\boldsymbol{k}}$ is $N \times N$ diagonal. $\mathcal{P}_{\boldsymbol{k}}$ is updated as follows:

1) Compute the entries of $\boldsymbol{\Omega}_{k+2}$ using the following equation:

$$
\boldsymbol{\Omega}_{k+2}=\left[\operatorname{diag}\left(\left|\mathbf{Y}^{(k)}\right|^{2}+\left|\mathbf{Y}^{(k+1)}\right|^{2}\right)^{-1}+\lambda^{-1} \mathbf{P}_{k}\right]^{-1}
$$

The matrix inversion needed to compute the entries of $\boldsymbol{\Omega}_{k+2}$ is simply $N$ scalar inversions since $\mathbf{P}_{\boldsymbol{k}}$ is diagonal.

2) Update $\mathbf{P}_{k+2}$ via

$$
\mathbf{P}_{k+2}=\lambda^{-1}\left[\mathbf{P}_{k}-\lambda^{-1} \mathbf{P}_{k} \boldsymbol{\Omega}_{k+2} \mathbf{P}_{k}\right]
$$

3) Repeat the previous steps for each iteration.

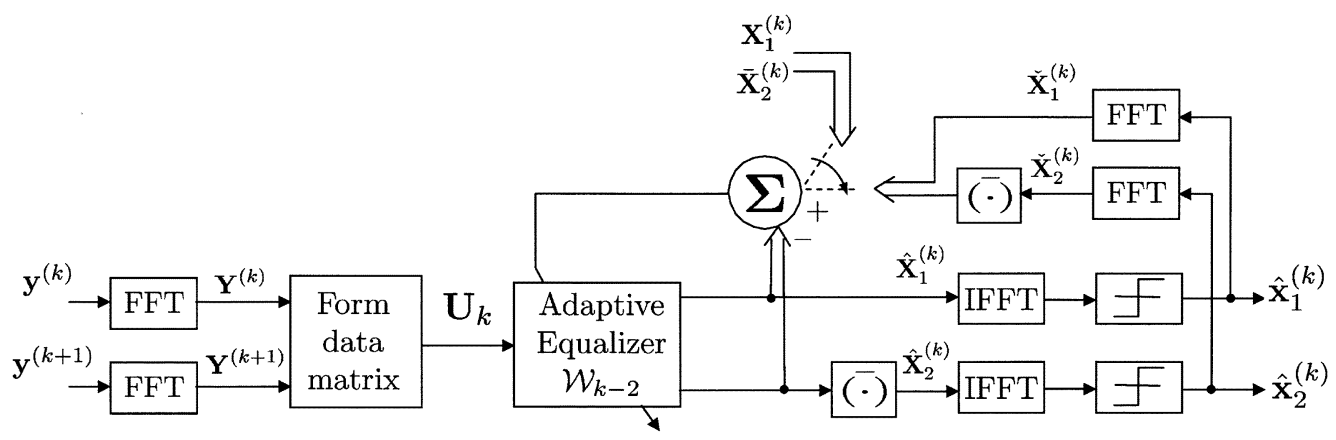

Fig. 6. Proposed receiver block diagram for single user with two-transmit one-receive antennas.

Then, $\mathcal{P}_{k+2}$ has the desired structure with

$$
\mathbf{P}_{k+2}=\lambda^{-1}\left(\mathbf{P}_{k}-\lambda^{-1} \mathbf{P}_{k} \boldsymbol{\Omega}_{k+2} \mathbf{P}_{k}\right)
$$

where

$$
\boldsymbol{\Omega}_{k+2}=\boldsymbol{\Theta}_{k+2} \operatorname{diag}\left(\left|\mathbf{Y}^{(k)}\right|^{2}+\left|\mathbf{Y}^{(k+1)}\right|^{2}\right) .
$$

Substituting (21) into (23), we get

$$
\boldsymbol{\Omega}_{k+2}=\left[\operatorname{diag}\left(\left|\mathbf{Y}^{(k)}\right|^{2}+\left|\mathbf{Y}^{(k+1)}\right|^{2}\right)^{-1}+\lambda^{-1} \mathbf{P}_{k}\right]^{-1} .
$$

Therefore, the algorithm collapses to

$$
\mathcal{W}_{k+2}=\mathcal{W}_{k}+\left(\begin{array}{cc}
\mathbf{P}_{k+2} & \mathbf{0} \\
\mathbf{0} & \mathbf{P}_{k+2}
\end{array}\right) \mathbf{U}_{k+2}^{*}\left[\mathbf{D}_{k+2}-\mathbf{U}_{k+2} \mathcal{W}_{k}\right]
$$

where $\mathbf{P}_{k+2}$ is $N \times N$ diagonal computed via (22) and (24). The procedure for updating the entries of $\mathcal{P}_{k+2}$ is shown in Table I. The block diagram of the resulting adaptive receiver is depicted in Fig. 6. The received signal is transformed to the frequency domain using FFT; then, the data matrix $\mathbf{U}_{k}$ in (16) is formed. The filter output is the product of the data matrix $\mathbf{U}_{k}$ and the filter coefficients $\mathcal{W}_{k-2}$. The filter output $\mathbf{U}_{k} \mathcal{W}_{k-2}$ is transformed back to the time domain using IFFT, and a decision device is used to generate the receiver output. The output of the equalizer is compared with the desired response to generate an error vector. The error vector is used to update the equalizer coefficients according to the RLS algorithm. The equalizer operates in a training mode until it converges, and then, it switches to a decision-directed mode, where previous decisions are used to update the equalizer coefficients for tracking. When tracking channels with fast variations, retraining blocks might be needed to prevent divergence of the adaptive algorithm.

\section{B. Two-User Transmissions}

Starting with (11), since $\Lambda_{11}$ and $\Lambda_{22}$ are Alamouti-like, we can easily verify that both $-\boldsymbol{\Lambda}_{12} \boldsymbol{\Lambda}_{22}^{-1}$ and $-\boldsymbol{\Lambda}_{21} \boldsymbol{\Lambda}_{11}^{-1}$ are also Alamouti-like. Combining the interference canceller and the MMSE equalizer, the equalizer output is then given by

$$
\begin{aligned}
\left(\begin{array}{l}
\hat{\mathbf{X}}_{1} \\
\hat{\mathbf{X}}_{2}
\end{array}\right) & =\left(\begin{array}{cc}
\tilde{\Sigma} \Sigma^{*} & 0_{2 N} \\
0_{2 N} & \tilde{\Delta} \Delta^{*}
\end{array}\right)\left(\begin{array}{cc}
\mathbf{I}_{2 N} & -\boldsymbol{\Lambda}_{12} \boldsymbol{\Lambda}_{22}^{-1} \\
-\boldsymbol{\Lambda}_{21} \boldsymbol{\Lambda}_{11}^{-1} & \mathbf{I}_{2 N}
\end{array}\right)\left(\begin{array}{l}
\mathbf{Y}_{1} \\
\mathbf{Y}_{2}
\end{array}\right) \\
& =\left(\begin{array}{cc}
\tilde{\boldsymbol{\Sigma}} \Sigma^{*} & -\tilde{\boldsymbol{\Sigma}} \boldsymbol{\Sigma}^{*} \boldsymbol{\Lambda}_{12} \boldsymbol{\Lambda}_{22}^{-1} \\
-\tilde{\Delta} \Delta^{*} \boldsymbol{\Lambda}_{21} \boldsymbol{\Lambda}_{11}^{-1} & \tilde{\Delta} \boldsymbol{\Delta}^{*}
\end{array}\right)\left(\begin{array}{l}
\mathbf{Y}_{1} \\
\mathbf{Y}_{2}
\end{array}\right) \\
& \triangleq\left(\begin{array}{ll}
\mathbf{A}_{11} & \mathbf{A}_{12} \\
\mathbf{A}_{21} & \mathbf{A}_{22}
\end{array}\right)\left(\begin{array}{l}
\mathbf{Y}_{1} \\
\mathbf{Y}_{2}
\end{array}\right)
\end{aligned}
$$


where $\tilde{\boldsymbol{\Sigma}}=\left(\boldsymbol{\Sigma}^{*} \boldsymbol{\Sigma}+\left(1 / \mathrm{SNR}_{1}\right) \mathbf{I}_{2 N}\right)^{-1}$ and $\tilde{\boldsymbol{\Delta}}=\left(\boldsymbol{\Delta}^{*} \boldsymbol{\Delta}+\right.$ $\left.\left(1 / \mathrm{SNR}_{2}\right) \mathbf{I}_{2 N}\right)^{-1}$ with $\mathrm{SNR}_{1}$ and $\mathrm{SNR}_{2}$ being the signal-tonoise ratios for the first user and the second user, respectively, and each $\mathbf{A}_{i j}, i, j=1,2$ has the following Alamouti-like structure:

$$
\mathbf{A}_{i j}=\left(\begin{array}{cc}
\mathbf{A}_{i j_{1}} & \mathbf{A}_{i j_{2}} \\
\mathbf{A}_{i j_{2}}^{*} & -\mathbf{A}_{i j_{1}}^{*}
\end{array}\right)
$$

Equation (26) can be rewritten alternatively as

$$
\begin{aligned}
\left(\begin{array}{c}
\hat{\mathbf{X}}_{11}^{(k)} \\
\hat{\mathbf{X}}_{1_{2}}^{(k)}
\end{array}\right)= & \left(\begin{array}{cc}
\operatorname{diag}\left(\mathbf{Y}_{1}^{(k)}\right) & \operatorname{diag}\left(\overline{\mathbf{Y}}_{1}^{(k+1)}\right) \\
-\operatorname{diag}\left(\mathbf{Y}_{1}^{(k+1)}\right) & \operatorname{diag}\left(\overline{\mathbf{Y}}_{1}^{(k)}\right)
\end{array}\right)\left(\begin{array}{l}
\mathbf{W}_{11_{1}} \\
\mathbf{W}_{11_{2}}
\end{array}\right) \\
& +\left(\begin{array}{cc}
\operatorname{diag}\left(\mathbf{Y}_{2}^{(k)}\right) & \operatorname{diag}\left(\overline{\mathbf{Y}}_{2}^{(k+1)}\right) \\
-\operatorname{diag}\left(\mathbf{Y}_{2}^{(k+1)}\right) & \operatorname{diag}\left(\overline{\mathbf{Y}}_{2}^{(k)}\right)
\end{array}\right)\left(\begin{array}{l}
\mathbf{W}_{12_{1}} \\
\mathbf{W}_{12_{2}}
\end{array}\right) \\
& \triangleq \mathbf{U}_{k}^{1} \mathcal{W}_{11}+\mathbf{U}_{k}^{2} \mathcal{W}_{12}=\left(\begin{array}{ll}
\mathbf{U}_{k}^{1} & \mathbf{U}_{k}^{2}
\end{array}\right)\left(\begin{array}{l}
\mathcal{W}_{11} \\
\mathcal{W}_{12}
\end{array}\right) \\
\triangleq & \mathcal{U}_{k} \mathcal{W}^{1}
\end{aligned}
$$

and

$$
\begin{aligned}
\left(\begin{array}{c}
\hat{\mathbf{X}}_{21}^{(k)} \\
\hat{\mathbf{X}}_{2_{2}}^{(k)}
\end{array}\right)= & \left(\begin{array}{cc}
\operatorname{diag}\left(\mathbf{Y}_{1}^{(k)}\right) & \operatorname{diag}\left(\overline{\mathbf{Y}}_{1}^{(k+1)}\right) \\
-\operatorname{diag}\left(\mathbf{Y}_{1}^{(k+1)}\right) & \operatorname{diag}\left(\overline{\mathbf{Y}}_{1}^{(k)}\right)
\end{array}\right)\left(\begin{array}{l}
\mathbf{W}_{21_{1}} \\
\mathbf{W}_{21_{2}}
\end{array}\right) \\
& +\left(\begin{array}{cc}
\operatorname{diag}\left(\mathbf{Y}_{2}^{(k)}\right) & \operatorname{diag}\left(\overline{\mathbf{Y}}_{2}^{(k+1)}\right) \\
-\operatorname{diag}\left(\mathbf{Y}_{2}^{(k+1)}\right) & \operatorname{diag}\left(\overline{\mathbf{Y}}_{2}^{(k)}\right)
\end{array}\right)\left(\begin{array}{l}
\mathbf{W}_{22_{1}} \\
\mathbf{W}_{22_{2}}
\end{array}\right) \\
& \triangleq \mathbf{U}_{k}^{1} \mathcal{W}_{21}+\mathbf{U}_{k}^{2} \mathcal{W}_{22}=\left(\begin{array}{ll}
\mathbf{U}_{k}^{1} & \mathbf{U}_{k}^{2}
\end{array}\right)\left(\begin{array}{l}
\mathcal{W}_{21} \\
\mathcal{W}_{22}
\end{array}\right) \\
& \triangleq \mathcal{U}_{k} \mathcal{W}^{2}
\end{aligned}
$$

where $\mathbf{W}_{i j_{1}}$ and $\mathbf{W}_{i j_{2}}$ are the vectors containing the diagonal elements of $\mathbf{A}_{i j_{1}}$ and $\mathbf{A}_{i j_{2}}$, respectively. Moreover, $\mathcal{W}_{i j}$ is a $2 N \times 1$ vector containing the elements of $\left\{\mathbf{W}_{i j_{1}}, \mathbf{W}_{i j_{2}}\right\}$, and $\mathbf{U}_{k}^{i}$ is an Alamouti-like matrix of size $2 N \times 2 N$ containing the received symbols at antenna $i$ from blocks $k$ and $k+1$. Equations (28) and (29) again reveal the special structure of the interference canceller for the STBC problem. In the nonadaptive scenario, the coefficients of $\mathcal{W}^{1}$ and $\mathcal{W}^{2}$ are calculated from a channel estimate at every block. Equations (28) and (29), on the other hand, indicate that $\mathcal{W}^{1}$ and $\mathcal{W}^{2}$ can be computed adaptively, e.g., by using RLS (for faster convergence). In this case, the receiver coefficients are updated every two blocks according to the recursions:

$$
\begin{aligned}
& \mathcal{W}_{k+2}^{1}=\mathcal{W}_{k}^{1}+\mathcal{P}_{k+2} \mathcal{U}_{k}^{*}\left[\mathbf{D}_{k+2}^{1}-\mathcal{U}_{k} \mathcal{W}_{k}^{1}\right] \\
& \mathcal{W}_{k+2}^{2}=\mathcal{W}_{k}^{2}+\mathcal{P}_{k+2} \mathcal{U}_{k}^{*}\left[\mathbf{D}_{k+2}^{2}-\mathcal{U}_{k} \mathcal{W}_{k}^{2}\right]
\end{aligned}
$$

where

$$
\mathcal{P}_{k+2}=\lambda^{-1}\left[\mathcal{P}_{k}-\lambda^{-1} \mathcal{P}_{k} \mathcal{U}_{k}^{*} \Pi(k) \mathcal{U}_{k} \mathcal{P}_{k}\right]
$$

and

$$
\Pi(k)=\left(\mathbf{I}_{2 N}+\lambda^{-1} \mathcal{U}_{k} \mathcal{P}_{k} \mathcal{U}_{k}^{*}\right)^{-1} .
$$

The initial conditions are $\mathcal{W}_{0}^{i}=0$ and $\mathcal{P}_{0}=\delta \mathbf{I}_{4 N}$, where $\delta$ is a large number, and $\mathbf{I}_{4 N}$ is the $4 N \times 4 N$ identity matrix. $\mathbf{D}_{k+2}^{i}$, $i=1, \cdots, 2$ is the desired response vector given by

$$
\mathbf{D}_{k+2}^{i}= \begin{cases}\left(\begin{array}{l}
\mathbf{X}_{i_{1}}^{(k+2)} \\
\overline{\mathbf{X}}_{i_{2}}^{(k+2)}
\end{array}\right), & \text { for training } \\
\left(\begin{array}{l}
\check{\mathbf{X}}_{i_{1}}^{(k+2)} \\
\overline{\mathbf{X}}_{i_{2}}^{(k+2)}
\end{array}\right), & \text { for decision-directed tracking. }\end{cases}
$$

Now, although matrix inversion is apparently needed in (32) for operation of the RLS algorithm, the computational complexity can be significantly reduced, and matrix inversion can be avoided altogether by exploiting the special STBC structure. Actually, the complexity of the algorithm can again be shown to be similar to that of an LMS implementation. The rationale behind complexity reduction is as follows. Starting with (31) at $k=0$, we get

$$
\mathcal{P}_{2}=\lambda^{-1}\left[\delta \mathbf{I}_{4 N}-\lambda^{-1} \delta^{2}\left(\begin{array}{c}
\mathbf{U}_{2}^{1 *} \\
\mathbf{U}_{2}^{2 *}
\end{array}\right) \bar{\Pi}(0)\left(\begin{array}{ll}
\mathbf{U}_{2}^{1} & \mathbf{U}_{2}^{2}
\end{array}\right)\right] .
$$

However, $\Pi(0)$ evaluates to

$$
\Pi(0)=\left(\mathbf{I}_{2 N}+\lambda^{-1} \delta\left(\mathbf{U}_{2}^{1} \mathbf{U}_{2}^{1 *}+\mathbf{U}_{2}^{2} \mathbf{U}_{2}^{2 *}\right)\right)^{-1}=\left(\begin{array}{cc}
\boldsymbol{\Pi}_{N} & \mathbf{0}_{N} \\
\mathbf{0}_{N} & \boldsymbol{\Pi}_{N}
\end{array}\right)
$$

where $\Pi(0)$ is $2 N \times 2 N$, and $\Pi_{N}$ is $N \times N$ diagonal and given by

$$
\begin{aligned}
\boldsymbol{\Pi}_{N}=\left(\mathbf{I}_{N}+\right. & \lambda^{-1}\left(\operatorname{diag}\left(\left|\mathbf{Y}_{1}^{0}\right|^{2}+\left|\mathbf{Y}_{1}^{1}\right|^{2}\right)\right. \\
& \left.\left.+\operatorname{diag}\left(\left|\mathbf{Y}_{2}^{0}\right|^{2}+\left|\mathbf{Y}_{2}^{1}\right|^{2}\right)\right)\right)^{-1}
\end{aligned}
$$

Substituting (35) into (34), we find that $\mathcal{P}_{2}$ is given by

$$
\begin{aligned}
\mathcal{P}_{2} & =\lambda^{-1} \delta \mathbf{I}_{4 N}-\left(\lambda^{-1} \delta\right)^{2} \cdot\left(\begin{array}{ll}
\mathbf{U}_{2}^{1 *} \boldsymbol{\Pi}(0) \mathbf{U}_{2}^{2} & \mathbf{U}_{2}^{1 *} \boldsymbol{\Pi}(0) \mathbf{U}_{2}^{2} \\
\mathbf{U}_{2}^{2 *} \boldsymbol{\Pi}(0) \mathbf{U}_{1}^{2} & \mathbf{U}_{2}^{2 *} \boldsymbol{\Pi}(0) \mathbf{U}_{2}^{2}
\end{array}\right) \\
& =\left(\begin{array}{ll}
\mathbf{P}_{11}(2) & \mathbf{P}_{12}(2) \\
\mathbf{P}_{21}(2) & \mathbf{P}_{22}(2)
\end{array}\right)
\end{aligned}
$$

where $\mathbf{P}_{11}(2)$ and $\mathbf{P}_{22}(2)$ are $2 N \times 2 N$ diagonal and $\mathbf{P}_{12}(2)$ and $\mathbf{P}_{21}(2)$ have an Alamouti-like structure. Proceeding for $k=$ 2 , we get

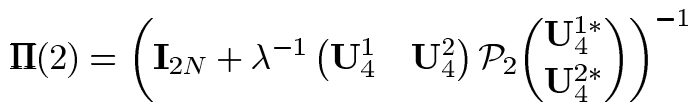

$$
\begin{aligned}
& =\left(\mathbf{I}_{2 N}+\lambda^{-1}\left(\mathbf{U}_{4}^{1} \mathbf{P}_{21} \mathbf{U}_{4}^{1 *}+\mathbf{U}_{4}^{2} \mathbf{P}_{23} \mathbf{U}_{4}^{2 *}\right.\right. \\
& \left.\left.+\mathbf{U}_{4}^{1} \mathbf{P}_{22} \mathbf{U}_{4}^{2 *}+\mathbf{U}_{4}^{2} \mathbf{P}_{24} \mathbf{U}_{4}^{2 *}\right)\right)^{-1} \text {. }
\end{aligned}
$$

After some simple algebra, it can be verified that $\Pi(2)$ has the form

$$
\boldsymbol{\Pi}(2)=\left(\begin{array}{ll}
\boldsymbol{\Pi}_{11}(2) & \boldsymbol{\Pi}_{12}(2) \\
\boldsymbol{\Pi}_{21}(2) & \boldsymbol{\Pi}_{22}(2)
\end{array}\right) \triangleq\left(\begin{array}{ll}
\boldsymbol{\Psi}_{11}(2) & \boldsymbol{\Psi}_{12}(2) \\
\boldsymbol{\Psi}_{21}(2) & \boldsymbol{\Psi}_{22}(2)
\end{array}\right)^{-1}
$$



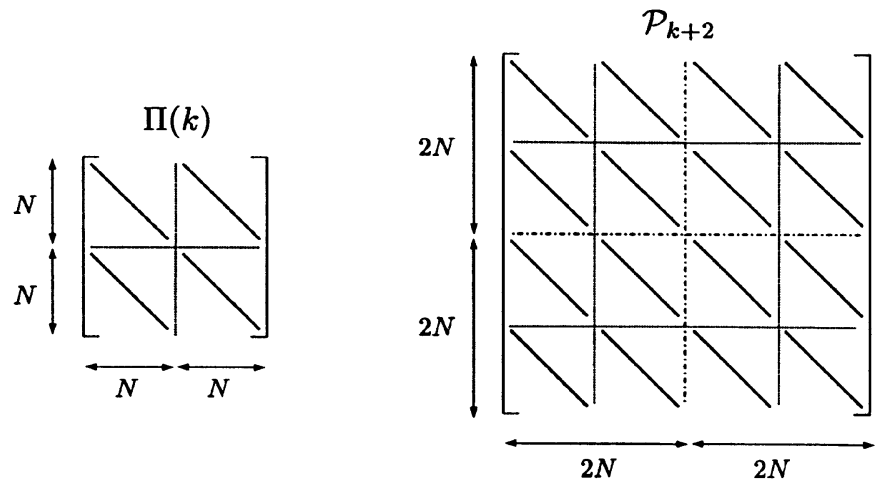

Fig. 7. Matrix structures of $\Pi(k)$ and $\mathcal{P}_{k+2}$ for $k \leq 2$.

where $\Pi_{i j}(2), i, j=1,2$ are $N \times N$ diagonal matrices. Using block matrix inversion [15], $\Pi(2)$ evaluates to (40), shown at the bottom of the page, where $\boldsymbol{\Sigma}_{\Psi}(2)=\boldsymbol{\Psi}_{11}(2)-\boldsymbol{\Psi}_{12}(2) \boldsymbol{\Psi}_{22}^{-1}(2) \boldsymbol{\Psi}_{21}(2)$ is the Schur complement of $\boldsymbol{\Psi}_{22}(2)$. Since $\boldsymbol{\Pi}_{i j}(2), i, j=1,2$ are all diagonal matrices, no matrix inversion is needed to calculate the matrix inverse in (40). Using (40), $\mathcal{P}_{4}$ is then found to be

$$
\mathcal{P}_{4}=\lambda^{-1} \mathcal{P}_{2}-\lambda^{-1} \mathcal{P}_{2} \mathcal{U}_{2}^{*} \Pi(2) \mathcal{U}_{2} \mathcal{P}_{2}
$$

It follows that $\mathcal{P}_{4}$ is a $4 N \times 4 N$ block matrix that consists of $16 N \times N$ diagonal matrices. This means that the number of entries to be calculated is much lower than for a full matrix. If we proceed further beyond $k=2$, we will find that the structures for $\Pi(k)$ and $\mathcal{P}_{k+2}$ stay the same. The matrix structures are shown in Fig. 7. Table II shows how we can use the structure of $\mathcal{P}_{k+2}$ to update its entries. It is worth mentioning that all $N \times N$ matrices in Table II are diagonal. This means that any matrix multiplication is simply evaluated by $N$ scalar multiplications. The structure of the receiver can bee seen in Fig. 8 for the case of $M=2$.

\section{Multiuser Transmissions}

By inspecting the structure of the interference canceller described in Section II, we find that it successively multiplies the received symbols of each user with Alamouti-like matrices in order to decouple them. We can verify that the overall response of the interference canceller and the MMSE equalizers, i.e., the mapping from the $\left\{\mathbf{Y}_{i}\right\}$ to the $\left\{\hat{\mathbf{X}}_{i}\right\}$ has the following form:

$$
\left(\begin{array}{c}
\hat{\mathbf{X}}_{1} \\
\hat{\mathbf{X}}_{2} \\
\vdots \\
\hat{\mathbf{X}}_{M}
\end{array}\right)=\left(\begin{array}{cccc}
\mathbf{A}_{11} & \mathbf{A}_{12} & \cdots & \mathbf{A}_{1 M} \\
\mathbf{A}_{21} & \mathbf{A}_{22} & \cdots & \mathbf{A}_{2 M} \\
\vdots & \vdots & \ddots & \vdots \\
\mathbf{A}_{M 1} & \mathbf{A}_{M 2} & \cdots & \mathbf{A}_{M M}
\end{array}\right)\left(\begin{array}{c}
\mathbf{Y}_{1} \\
\mathbf{Y}_{2} \\
\vdots \\
\mathbf{Y}_{M}
\end{array}\right)
$$

where each $\mathbf{A}_{i j}, i, j=1, \cdots, M$ has an Alamouti-like structure given by (27). For the two-user case, the entries of $\mathbf{A}_{i j}, i, j=1$,
2 are given explicitly in (26). However, the explicit knowledge of the entries of these matrices is not needed for the development of the adaptive solution. The adaptive solution proposed here works for any $\mathbf{A}_{i j}^{\prime} s$, regardless of their entries, as long as they have an Alamouti-like structure. Equation (42) can be rewritten as

$$
\begin{aligned}
\left(\begin{array}{c}
\hat{\mathbf{X}}_{i_{1}}^{(k)} \\
\overline{\hat{\mathbf{X}}}_{i_{2}}^{(k)}
\end{array}\right) & =\sum_{j=1}^{M}\left(\begin{array}{cc}
\operatorname{diag}\left(\mathbf{Y}_{j}^{(k)}\right) & \operatorname{diag}\left(\overline{\mathbf{Y}}_{j}^{(k+1)}\right) \\
\operatorname{diag}\left(\mathbf{Y}_{j}^{(k+1)}\right) & -\operatorname{diag}\left(\overline{\mathbf{Y}}_{j}^{(k)}\right)
\end{array}\right)\left(\begin{array}{l}
\mathbf{W}_{i j_{1}} \\
\mathbf{W}_{i j_{2}}
\end{array}\right) \\
& \triangleq \sum_{j=1}^{M} \mathbf{U}_{k}^{j} \mathcal{W}_{i j} \\
& =\left(\mathbf{U}_{k}^{1} \ldots \mathbf{U}_{k}^{M}\right)\left(\begin{array}{c}
\mathcal{W}_{i 1} \\
\vdots \\
\mathcal{W}_{i M}
\end{array}\right)=\mathcal{U}_{k} \mathcal{W}^{i}
\end{aligned}
$$

where $i=1 \cdots M$. Equation (43) also indicates that the receiver coefficients can be computed adaptively. We use the RLS algorithm to update the receiver coefficients every two blocks according to the recursions

$\mathcal{W}_{k+2}^{i}=\mathcal{W}_{k}^{i}+\mathcal{P}_{k+2} \mathcal{U}_{k+2}^{*}\left[\mathbf{D}_{k+2}^{i}-\mathcal{U}_{k} \mathcal{W}_{k}^{i}\right], \quad i=1, \cdots, M$

where

$$
\mathcal{P}_{k+2}=\lambda^{-1}\left[\mathcal{P}_{k}-\lambda^{-1} \mathcal{P}_{k} \mathcal{U}_{k+2}^{*} \Pi(k) \mathcal{U}_{k+2} \mathcal{P}_{k}\right]
$$

and

$$
\Pi(k)=\left(\mathbf{I}_{2 N}+\lambda^{-1} \mathcal{U}_{k+2} \mathcal{P}_{k} \mathcal{U}_{k+2}^{*}\right)^{-1} .
$$

The initial conditions are $\mathcal{W}_{0}^{i}=\mathbf{0}$ and $\mathcal{P}_{0}=\delta \mathbf{I}_{2 M N}, \delta$ is a large number, and $\mathbf{I}_{2 M N}$ is the $2 M N \times 2 M N$ identity matrix. $\mathbf{D}_{k+2}^{i}$ is the desired response vector given by (33). The block diagram of the adaptive receiver is shown in Fig. 8. The received signals from both antennas are transformed to the frequency domain using FFT, and then, the data matrices $\mathbf{U}_{k}^{1}, \cdots, \mathbf{U}_{k}^{M}$ in (43) are formed and passed through the filters to form the frequency domain estimates for the two users' transmitted data $\hat{\mathbf{X}}_{1}, \cdots, \hat{\mathbf{X}}_{M}$. These outputs are transformed back to the time domain using IFFT, and decision devices are used to generate the receiver outputs. The receiver operates in a training mode where known training data are used to generate the error vectors and update the receiver coefficients until they converge; then, it switches to a decision-directed mode, where previous decisions are used to update the receiver coefficients for tracking. For decision-directed operation, the reconstructed data are transformed back to frequency domain and compared with the corresponding receiver outputs to generate error vectors. The error vectors are used to update the coefficients according to the RLS algorithm.

$$
\Pi(2)=\left(\begin{array}{cc}
\boldsymbol{\Sigma}_{\boldsymbol{\Psi}}^{-1}(2) & \boldsymbol{\Sigma}_{\boldsymbol{\Psi}}^{-1}(2) \boldsymbol{\Psi}_{12}(2) \boldsymbol{\Psi}_{22}^{-1}(2) \\
\boldsymbol{\Psi}_{22}^{-1}(2) \boldsymbol{\Psi}_{21}(2) \boldsymbol{\Sigma}_{\boldsymbol{\Psi}}^{-1}(2) & \boldsymbol{\Psi}_{22}^{-1}(2)+\boldsymbol{\Psi}_{22}^{-1}(2) \boldsymbol{\Psi}_{21}(2) \boldsymbol{\Sigma}_{\boldsymbol{\Psi}}^{-1}(2) \boldsymbol{\Psi}_{12}(2) \boldsymbol{\Psi}_{22}^{-1}(2)
\end{array}\right)
$$


TABLE II

Define

$$
\mathcal{P}_{k} \triangleq\left(\begin{array}{llll}
\mathbf{P}_{11}(k) & \mathbf{P}_{12}(k) & \mathbf{P}_{13}(k) & \mathbf{P}_{14}(k) \\
\mathbf{P}_{21}(k) & \mathbf{P}_{22}(k) & \mathbf{P}_{23}(k) & \mathbf{P}_{24}(k) \\
\mathbf{P}_{31}(k) & \mathbf{P}_{32}(k) & \mathbf{P}_{33}(k) & \mathbf{P}_{34}(k) \\
\mathbf{P}_{41}(k) & \mathbf{P}_{42}(k) & \mathbf{P}_{43}(k) & \mathbf{P}_{44}(k)
\end{array}\right)
$$

where each $\mathbf{P}_{i j}(k)$ is $N \times N$ diagonal. Let

$$
\mathcal{U}_{k} \triangleq\left(\begin{array}{ll}
\mathbf{U}_{k}^{1} & \mathbf{U}_{k}^{2}
\end{array}\right)=\left(\begin{array}{llll}
\mathrm{U}_{11}(k) & \mathrm{U}_{21}(k) & \mathrm{U}_{31}(k) & \mathrm{U}_{41}(k) \\
\mathrm{U}_{12}(k) & \mathrm{U}_{22}(k) & \mathrm{U}_{32}(k) & \mathrm{U}_{42}(k)
\end{array}\right)
$$

where the entries of $\mathcal{U}_{k}$ are given by Equation (28). Also, each $\mathrm{U}_{i j}(k)$ is $N \times N$ diagonal. $\mathcal{P}_{k}$ is now updated as follows:

1) Let

$$
\boldsymbol{\Psi}_{k}=\mathbf{I}_{2 N}+\lambda^{-1} \mathbf{U}_{k+2} \mathcal{P}_{k} \mathbf{U}_{k+2}^{*}=\left(\begin{array}{ll}
\boldsymbol{\Psi}_{11}(k) & \boldsymbol{\Psi}_{12}(k) \\
\boldsymbol{\Psi}_{21}(k) & \boldsymbol{\Psi}_{22}(k)
\end{array}\right)
$$

2) Compute its block entries $\Psi_{i j}(k), i, j=1,2$, as follows

$$
\Psi_{i j}(k)=\xi_{i j}+\lambda^{-1} \cdot \sum_{l=1}^{4} \sum_{m=1}^{4} \mathbf{U}_{m i}(k+2) \mathbf{P}_{m l}(k) \mathbf{U}_{l j}^{*}(k+2)
$$

where $\xi_{i j}=\mathrm{I}_{N}$ when $i=j$, and zero otherwise. Again, each $\boldsymbol{\Psi}_{i j}(k)$ is $N \times N$ diagonal.

3) Compute

$$
\Pi(k)=\left(\begin{array}{cc}
\Sigma_{\Psi}^{-1}(k) & \Sigma_{\Psi}^{-1}(k) \Psi_{12} \Psi_{22}^{-1} \\
\Psi_{22}^{-1} \Psi_{21} \Sigma_{\Psi}^{-1}(k) & \Psi_{22}^{-1}+\Psi_{22}^{-1} \Psi_{21} \Sigma_{\Psi}^{-1}(k) \Psi_{12} \Psi_{22}^{-1}
\end{array}\right)
$$

where $\boldsymbol{\Sigma}_{\Psi}(k)=\Psi_{11}(k)-\Psi_{12}(k) \Psi_{22}^{-1}(k) \Psi_{21}(k)$ is the Schur complement of $\Psi_{22}(k)$ and the time index $(k)$ has been dropped for compactness. The $\Pi_{i j}(k)$ blocks of $\Pi(k)$ are also $N \times N$ diagonal.

4) Define $\Phi_{k}=\mathcal{U}_{k+2}^{*} \Pi(k) \mathcal{U}_{k+2}$. It has a structure similar to $\mathcal{P}_{k}$, then the $N \times N$ diagonal matrices $\Phi_{i j}(k)$, $i, j=1 \cdots 4$, are given by

$$
\boldsymbol{\Phi}_{i j}(k)=\sum_{l=1}^{2} \sum_{m=1}^{2} \mathbf{U}_{i l}^{*}(k+2) \boldsymbol{\Pi}_{l m}(k) \mathbf{U}_{j m}(k+2)
$$

5) Update $\mathbf{P}_{i j}(k+2)$ as

$$
\mathbf{P}_{i j}(k+2)=\lambda^{-1} \mathbf{P}_{i j}(k)-\lambda^{-2} \sum_{l=1}^{4} \sum_{m=1}^{4} \mathbf{P}_{i m}(k) \Phi_{m l}(k) \mathbf{P}_{l j}(k)
$$

6) Repeat the previous steps for each iteration.

Again, the computational complexity can be significantly reduced, and matrix inversion can be avoided by exploiting the special STBC structure, in a manner similar to what we did in the two-user case in the previous section. We briefly explain the rationale behind complexity reduction. Starting with (45) at $k=0$, we get

$$
\mathcal{P}_{2}=\lambda^{-1}\left[\delta \mathbf{I}_{2 M N}-\lambda^{-1} \delta^{2} \mathcal{U}_{2}^{*} \Pi(0) \mathcal{U}_{2}\right]
$$

However, $\boldsymbol{\Pi}(0)$ evaluates to

$$
\boldsymbol{\Pi}(0)=\left(\mathbf{I}_{2 N}+\lambda^{-1} \delta \mathcal{U}_{2} \mathcal{U}_{2}^{*}\right)^{-1}=\left(\begin{array}{cc}
\boldsymbol{\Pi}_{N} & \mathbf{0}_{N} \\
\mathbf{0}_{N} & \boldsymbol{\Pi}_{N}
\end{array}\right)
$$

where $\Pi_{N}$ is $N \times N$ diagonal and given by

$$
\mathbf{\Pi}_{N}=\left(\mathbf{I}_{N}+\lambda^{-1} \delta \sum_{i=1}^{M} \operatorname{diag}\left(\left|\mathbf{Y}_{i}^{0}\right|^{2}+\left|\mathbf{Y}_{i}^{1}\right|^{2}\right)\right)^{-1} .
$$




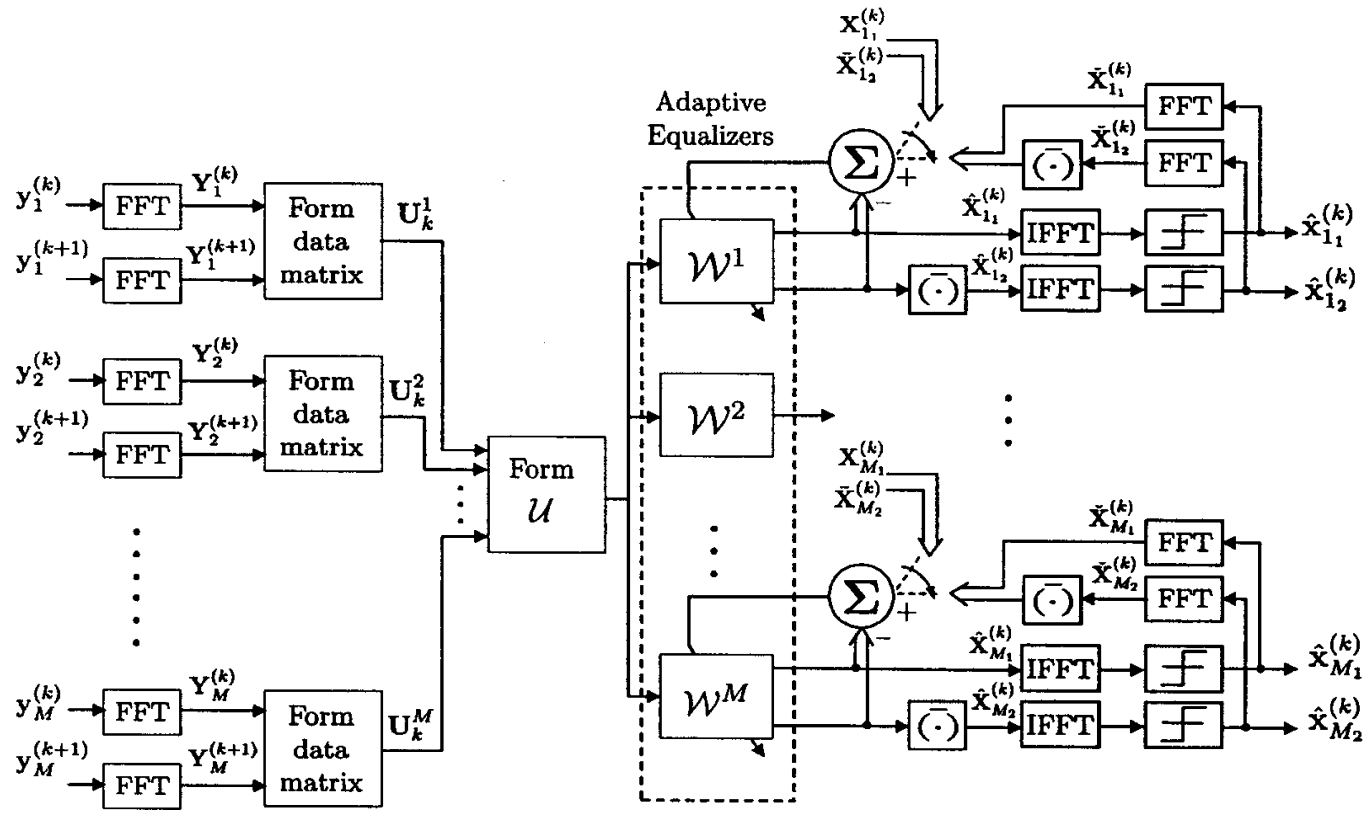

Fig. 8. Adaptive receiver structure for an $M$-user system with $2 M$-transmit and $M$-receive antennas.

Substituting (48) into (47), we find that $\mathcal{P}_{2}$ is given by

$$
\begin{aligned}
\mathcal{P}_{2} & =\lambda^{-1} \delta \mathbf{I}_{2 M N}-\left(\lambda^{-1} \delta\right)^{2} \mathcal{U}_{2}^{*} \boldsymbol{\Pi}(0) \mathcal{U}_{2} \\
& =\left(\begin{array}{ccc}
\mathbf{P}_{11}(2) & \ldots & \mathbf{P}_{12 M}(2) \\
\vdots & \ddots & \vdots \\
\mathbf{P}_{2 M 1}(2) & \ldots & \mathbf{P}_{2 M 2 M}(2)
\end{array}\right)
\end{aligned}
$$

where $\mathbf{P}_{i j}(2), i, j=1 \cdots 2 M$ are $N \times N$ diagonal. Proceeding for $k=2$, it can be verified that $\Pi(2)$ has the same form as (39); then, its entries can be computed using (40). $\mathcal{P}_{4}$ is then found to be

$$
\mathcal{P}_{4}=\lambda^{-1}\left[\mathcal{P}_{2}-\lambda_{2}^{-1} \mathcal{P}_{2} \mathcal{U}_{4}^{*} \Pi(2) \mathcal{U}_{4} \mathcal{P}_{2}\right]
$$

It follows that $\mathcal{P}_{4}$ is a $2 M N \times 2 M N$ block matrix that consists of $4 M^{2} N \times N$ diagonal matrices. If we proceed further beyond $k=2$, we will find that the structures for $\Pi(k)$ and $\mathcal{P}_{k+2}$ stay the same. Table III shows how we can use the structure of $\mathcal{P}_{k+2}$ to update its entries. Again, all $N \times N$ matrices in Table III are diagonal. This means that any matrix multiplication is simply evaluated by $N$ scalar multiplications.

\section{Adaptation for Slowly Varying Channels}

The adaptive algorithm in the multiuser case can be further simplified without much loss in performance for slowly varying channels by forcing $\mathcal{P}_{k}$ to be a diagonal matrix. In this way, the RLS algorithm update (44) can be written as

$$
\mathcal{W}_{k+2}^{i}=\mathcal{W}_{k}^{i}+\left(\begin{array}{ccc}
\mathcal{P}_{k+2}^{1} & & \\
& \ddots & \\
& & \mathcal{P}_{k+2}^{M}
\end{array}\right) \mathcal{U}_{k+2}^{*}\left[\mathbf{D}_{k+2}^{i}-\mathcal{U}_{k} \mathcal{W}_{k}^{i}\right]
$$

for $i=1, \cdots, M$. In the light of (43), we can split (51) into $M$ independent equations given by

$\mathcal{W}_{i j}(k+2)=\mathcal{W}_{i j}(k)+\mathcal{P}_{k+2}^{j} \mathbf{U}_{k+2}^{j^{*}}\left[\mathbf{D}_{k+2}^{i}-\sum_{l=1}^{M} \mathbf{U}_{k}^{l} \mathcal{W}_{i l}(k)\right]$

where $i, j=1, \cdots, M$. Now, we have $M^{2}$ filters $\mathcal{W}_{i j}, i, j=$ $1, \cdots, M$ adapted individually by means of the RLS equations

$$
\begin{aligned}
& \mathcal{W}_{i j}(k+2)=\mathcal{W}_{i j}(k)+\mathcal{P}_{k+2}^{j} \mathbf{U}_{k+2}^{j^{*}} \\
& \quad \times\left[\left(\mathbf{D}_{k+2}^{i}-\mathbf{U}_{k}^{j} \mathcal{W}_{i j}(k)\right)-\sum_{\substack{l=1 \\
l \neq j}}^{M} \mathbf{U}_{k}^{l} \mathcal{W}_{i l}(k)\right]
\end{aligned}
$$

where now

$$
\begin{aligned}
& \mathcal{P}_{k+2}^{j}=\lambda^{-1}\left[\mathcal{P}_{k}^{j}-\lambda^{-1} \mathcal{P}_{k}^{j} \mathbf{U}_{k+2}^{j^{*}}\right. \\
&\left.\cdot\left(\mathbf{I}_{2 N}+\lambda^{-1} \mathbf{U}_{k+2}^{j} \mathcal{P}_{k}^{j} \mathbf{U}_{k+2}^{j^{*}}\right)^{-1} \mathbf{U}_{k+2}^{j} \mathcal{P}_{k}^{j}\right]
\end{aligned}
$$

for $j=1, \cdots, M$. In this case, RLS uses a modified error signal. The modified error signal for each filter depends on the outputs of $M-1$ other filters. Due to the fact that the filter outputs are far from the correct values at the beginning of the receiver operation, the algorithm is expected to have slower convergence than the one given by (44). However, when the channel is slowly varying, the algorithm can track channel variations effectively. For cases when the channel changes rapidly, the first algorithm given by (44) has superior performance. By comparing this case with the single-user case, we notice that $\mathcal{P}_{k+2}^{j}, j=1, \cdots, M$ has a structure similar to that of $\mathcal{P}_{k+2}$ in (19). Then, it can be computed as follows.

$$
\mathcal{P}_{k+2}^{j}=\left(\begin{array}{cc}
\mathbf{P}_{k+2}^{j} & \mathbf{0} \\
\mathbf{0} & \mathbf{P}_{k+2}^{j}
\end{array}\right)
$$


Define

$$
\mathcal{P}_{k} \triangleq\left(\begin{array}{cccc}
\mathbf{P}_{11}(k) & \mathbf{P}_{12}(k) & \ldots & \mathbf{P}_{12 M}(k) \\
\mathbf{P}_{21}(k) & \mathbf{P}_{22}(k) & \ldots & \mathbf{P}_{22 M}(k) \\
\vdots & \vdots & \ddots & \vdots \\
\mathbf{P}_{2 M 1}(k) & \mathbf{P}_{2 M 2}(k) & \ldots & \mathbf{P}_{2 M 2 M}(k)
\end{array}\right)
$$

where each $\mathbf{P}_{i j}(k)$ is $N \times N$ diagonal. Let

$$
\begin{aligned}
\mathcal{U}_{k} & \triangleq\left(\begin{array}{llll}
\mathbf{U}_{k}^{1} & \mathbf{U}_{k}^{2} & \ldots & \mathbf{U}_{k}^{M}
\end{array}\right) \\
& =\left(\begin{array}{llll}
\mathbf{U}_{11}(k) & \mathbf{U}_{21}(k) & \ldots & \mathbf{U}_{2 M_{1}}(k) \\
\mathbf{U}_{12}(k) & \mathbf{U}_{22}(k) & \ldots & \mathbf{U}_{2 M_{2}}(k)
\end{array}\right)
\end{aligned}
$$

where the entries of $\mathcal{U}_{k}$ are given by Equation (28). Also, each $U_{i j}(k)$ is $N \times N$ diagonal. $\mathcal{P}_{k}$ is now updated as follows:

1) Let

$$
\Psi_{k}=I_{2 N}+\lambda^{-1} \mathcal{U}_{k+2} \mathcal{P}_{k} \mathcal{U}_{k+2}^{*}=\left(\begin{array}{ll}
\Psi_{11}(k) & \Psi_{12}(k) \\
\Psi_{21}(k) & \Psi_{22}(k)
\end{array}\right)
$$

2) Compute its block entries $\Psi_{i j}(k), i, j=1,2$, as follows

$$
\Psi_{i j}(k)=\xi_{i j}+\lambda^{-1} \cdot \sum_{l=1}^{2 M} \sum_{m=1}^{2 M} \mathbf{U}_{m i}(k+2) \mathbf{P}_{m l}(k) \mathbf{U}_{l j}^{*}(k+2)
$$

where $\xi_{i j}=\mathbf{I}_{N}$ when $i=j$, and zero otherwise. Again, each $\Psi_{i j}(k)$ is $N \times N$ diagonal.

3) Compute

$$
\Pi(k)=\left(\begin{array}{cc}
\Sigma_{\Psi}^{-1}(k) & \Sigma_{\Psi}^{-1}(k) \Psi_{12} \Psi_{22}^{-1} \\
\Psi_{22}^{-1} \Psi_{21} \Sigma_{\Psi}^{-1}(k) & \Psi_{22}^{-1}+\Psi_{22}^{-1} \Psi_{21} \Sigma_{\Psi}^{-1}(k) \Psi_{12} \Psi_{22}^{-1}
\end{array}\right)
$$

where $\Sigma_{\Psi}(k)=\Psi_{11}(k)-\Psi_{12}(k) \Psi_{22}^{-1}(k) \Psi_{21}(k)$ is the Schur complement of $\Psi_{22}(k)$ and the time index (k) has been dropped for compactness. The $\Pi_{i j}(k)$ blocks of $\Pi(k)$ are also $N \times N$ diagonal.

4) Define $\Phi_{k}=\mathcal{U}_{k+2}^{*} \Pi(k) \mathcal{U}_{k+2}$. It has a structure similar to $\mathcal{P}_{k}$, then the $N \times N$ diagonal matrices $\boldsymbol{\Phi}_{i j}(k)$, $i, j=1 \cdots 2 M$, are given by

$$
\boldsymbol{\Phi}_{i j}(k)=\sum_{l=1}^{2} \sum_{m=1}^{2} \mathbf{U}_{i l}^{*}(k+2) \boldsymbol{\Pi}_{l m}(k) \mathbf{U}_{j m}(k+2)
$$

5) Update $\mathbf{P}_{i j}(k+2)$ as

$$
\mathbf{P}_{i j}(k+2)=\lambda^{-1} \mathbf{P}_{i j}(k)-\lambda^{-2} \sum_{l=1}^{2 M} \sum_{m=1}^{2 M} \mathbf{P}_{i m}(k) \Phi_{m l}(k) \mathbf{P}_{l j}(k)
$$

6) Repeat the previous steps for each iteration.

where

and

$\mathbf{P}_{k+2}^{j}=\lambda^{-1}\left[\mathbf{P}_{k}^{j}-\lambda^{-1} \mathbf{P}_{k}^{j} \mathbf{\Omega}_{k+2}^{j} \mathbf{P}_{k}^{j}\right]$

$\mathbf{\Omega}_{k+2}^{j}=\left[\operatorname{diag}\left(\left|\mathbf{Y}_{j}^{k}\right|^{2}+\left|\mathbf{Y}_{j}^{k+1}\right|^{2}\right)^{-1}+\lambda^{-1} \mathbf{P}_{k}^{j}\right]^{-1}$ 


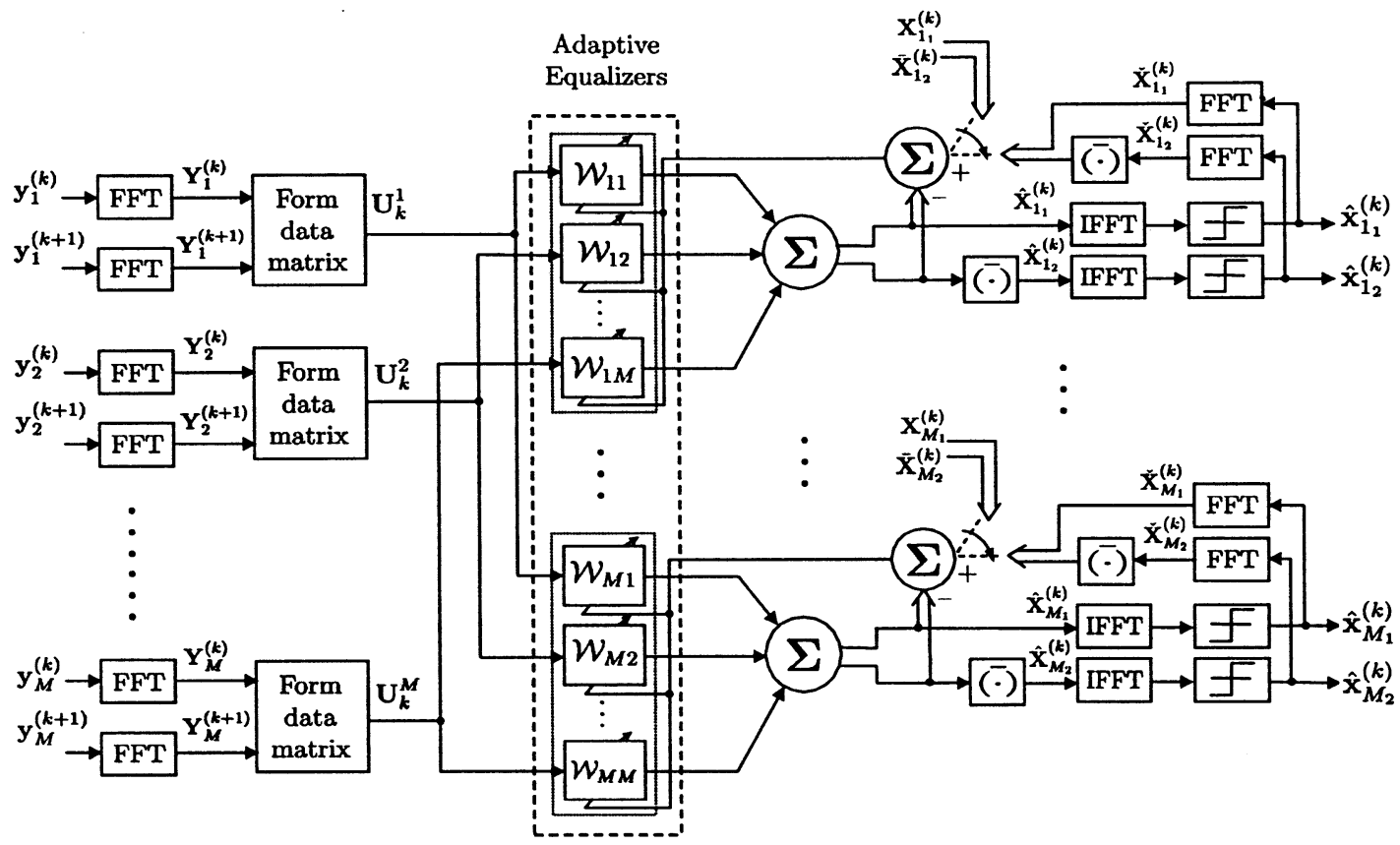

Fig. 9. Simplified adaptive receiver block diagram for multi-user transmissions.

Therefore, $\mathbf{P}_{k+2}^{j}$ is diagonal as computed via (55) and (56). The block diagram of the adaptive receiver with simplified RLS is shown in Fig. 9.

\section{E. Effect of Parameters Selection}

Different parameters affect the performance of the adaptive receivers. A judicious selection of these parameters is needed to achieve best performance. The main factor affecting the parameter selection is how fast the channel changes, which is measured by the Doppler frequency. As the Doppler frequency increases, the channel changes significantly even within one block, which violates the assumption that the channel is fixed over two consecutive blocks. In this case, shorter data blocks have to be used for better algorithm tracking. However, using smaller data blocks result in increased system overhead due to the cyclic prefix attached to each block. It is also useful to use more frequent retraining to prevent divergence although it increases the system overhead as well. Another parameter is the RLS forgetting factor $(\lambda)$. By using smaller $\lambda$, we decrease the algorithm memory and allow faster tracking. Careful selection of $\lambda$ must be used since RLS has numerical problems when $\lambda$ is reduced. To reduce system overhead, we can use smaller blocks for training than the actual data blocks. However, the number of equalizer coefficients depend on the block size. We need to calculate the remaining equalizer coefficients (the difference between date block size and training block size) by interpolation. It was shown in [12] that smaller training blocks can reduce the system overhead at the expense of a minor loss of performance. The effect of different parameters on the receiver performance is investigated in Section IV.

\section{Simulation Results}

In this section, we provide simulation results for the performance of the adaptive receivers for STBC. We simulated three different scenarios including one, two, and three users. In our simulations, we assumed a symbol rate of $271 \mathrm{kSymbols} / \mathrm{s}$. A typical urban (TU) channel is considered with a linearized GMSK transmit pulse shape. Furthermore, all channels are assumed to be independent. The overall channel impulse response memory of the channel is $\nu=3$. We start with the single-user case. Two transmit antennas with 8-PSK signal constellation are used. Data blocks of 32 symbols plus three symbols for the cyclic prefix are used. In the following figures, we focus on the performance of the RLS algorithm and how it is affected by different parameters. Fig. 10 shows the performance of the RLS algorithm at different Doppler frequencies. One block of training data is used every 50 blocks to prevent the divergence of the algorithm at high Doppler frequencies. It is obvious that the BER increases with Doppler frequency as a result of the inability of the algorithm to track the faster channel variations. Since the equalizer coefficients are updated on block-by-block basis, it is useful to use smaller data blocks. Retraining more often can also be a good solution. The effect of the block size and the number of blocks before retraining on the BER performance is shown in Fig. 11. It shows that retraining more often and using smaller data blocks can help improve the BER performance as the Doppler frequency increases. Despite the increase of the system overhead, dramatic BER reduction was observed as we used 16-symbol data blocks and as we used one retraining block every 25 data blocks.

In the two-user and three-user scenarios, the receiver is equipped with two and three receive antennas, respectively. Each user is equipped with two transmit antennas, and 8-PSK signal constellation is used. Signal-to-interference noise ratio (SINR) is set to $0 \mathrm{~dB}$, i.e., both users are transmitting at the same power. Data blocks of 32 symbols plus three symbols for the cyclic prefix are used. Fig. 12 shows the bit-error-rate performance of the two-user and three-user systems compared 


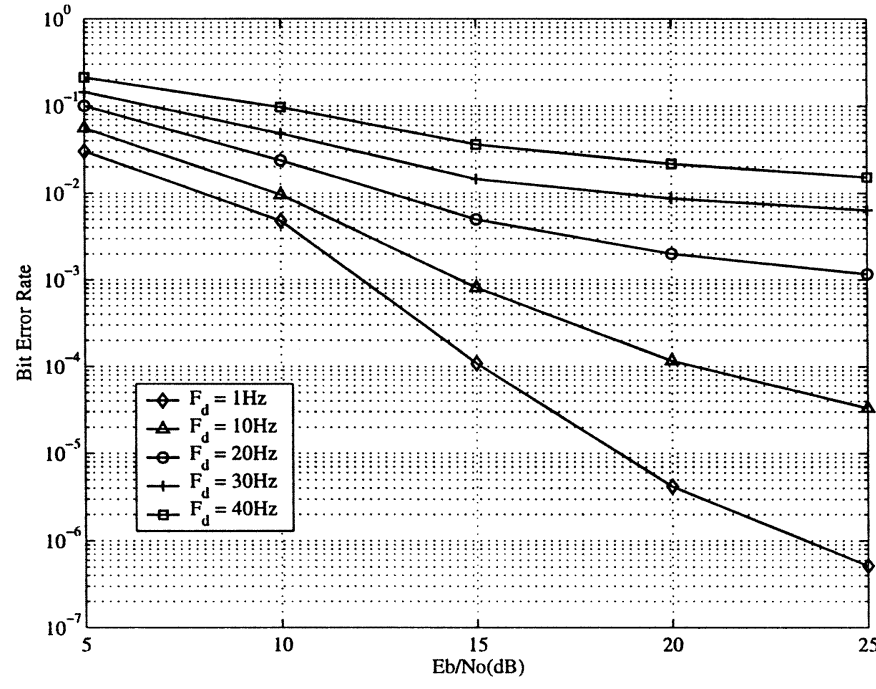

Fig. 10. Effect of Doppler frequency on the performance of RLS algorithm.

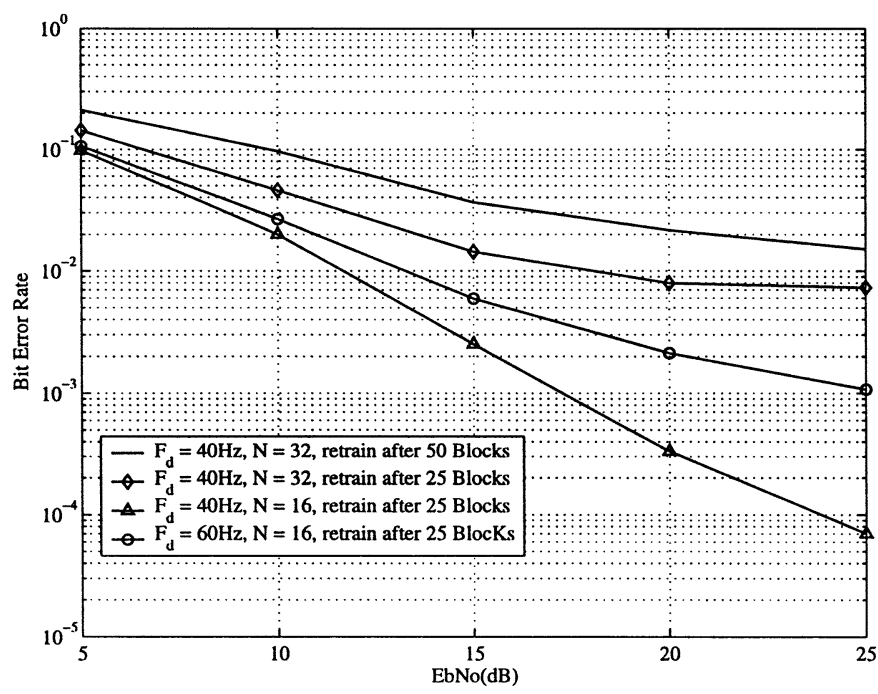

Fig. 11. Effect of block size and retraining frequency on tracking performance.

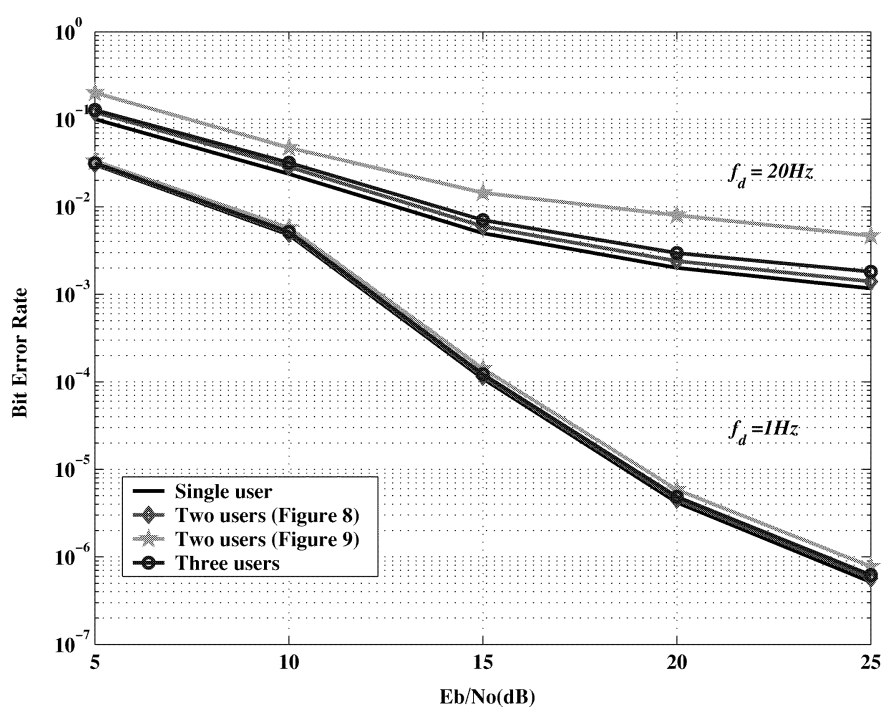

Fig. 12. BER performance of the adaptive receiver for two and three users. with the single-user case at two different Doppler frequencies. From this figure, it is clear that the adaptive interference cancellation technique can separate co-channel users without sacrificing performance. However, at higher Doppler frequencies, the scheme of Fig. 8 has a better performance than the one of Fig. 9. At very high Doppler frequencies, the RLS algorithm might not be able to track the channel variations. In this case, training more often can improve the system performance at the expense of increasing system overhead.

\section{CONCLUSIONS}

Adaptive receivers for space-time block-coded transmissions are developed for single-user, two-user, and multiuser transmissions. These receivers do not require explicit channel knowledge or channel estimation at the receiver. The receivers are based on a modified low-complexity RLS algorithm that exploits the rich structure of STBC to reduce the computational complexity to that of LMS algorithm. Moreover, they are capable of separating $M$ co-channel users without sacrificing performance or bandwidth. Both training and tracking performance results in a time-varying frequency-selective fading environment are presented. The effect of different design parameters on the performance of the receivers is discussed. Different techniques for performance improvement and computational complexity and system overhead reduction by a better utilization of the receivers'parameters are also suggested. In a related recent work [16], it is shown that the complexity of the receivers can be further simplified by performing the equalization at a per-frequency-bin level rather than at a block level. in this way, the complexity of the implementation is reduced to $\mathcal{O}\left(N M^{2}\right)$, where $M$ is the number of users, and $N$ is the block size.

\section{REFERENCES}

[1] A. F. Naguib, N. Seshadri, and A. R. Calderbank, "Increasing data rate over wireless channels: space-time coding and signal processing for high data rate wireless communications," IEEE Commun. Mag., vol. 17, pp. 76-92, May 2000.

[2] J. H. Winters, J. Salz, and R. D. Gitlin, "The impact of antenna diversity on the capacity of wireless communication systems," IEEE Trans. Commun., vol. 42, pp. 1740-1751, Feb. 1994.

[3] A. F. Naguib, N. Seshadri, and A. R. Calderbank, "Applications of space-time block codes and interference suppression for high capacity and high data rate wireless systems," in Proc. 32nd Asilomar Conf. Signals, Syst., Comput., Pacific Grove, CA, Nov. 1998, pp. 1803-1810.

[4] S. Alamouti, "A simple transmit diversity technique for wireless communications," IEEE J. Select. Areas Commun., vol. 16, pp. 1451-1458, Oct. 1998.

[5] A. Stamoulis, N. Al-Dhahir, and A. R. Calderbank, "Further results on interference cancellation and space-time block codes," in Proc. 35th Asilomar Conf. Signals, Syst., Comput., Pacific Grove, CA, Nov. 2001, pp. 257-261.

[6] E. Lindskog and A. Paulraj, "A transmit diversity scheme for delay spread channels," in Proc. Int. Contr. Conf., New Orleans, LA, June 2000, pp. 307-311.

[7] Z. Liu, G. Giannakis, A. Scaglione, and S. Barbarossa, "Decoding and equalization of unknown multipath channels based on block precoding and transmit antenna diversity," in Proc. 33rd Asilomar Conf. Signals, Syst., Comput., Pacific Grove, CA, Nov. 1999, pp. 1557-1561.

[8] N. Al-Dhahir, "Single-carrier frequency-domain equalization for space-time block-coded transmissions over frequency-selective fading channels," IEEE Commun. Lett., vol. 5, pp. 304-306, July 2001.

[9] A. Czylwik, "Comparison between adaptive OFDM and single carrier modulation with frequency domain equalization," in Proc. Veh. Technol. Conf., New York, May 1997, pp. 865-869. 
[10] A. Oppenheim and R. Schafer, Discrete Time Signal Processing. Englewood Cliffs, NJ: Prentice-Hall, 1989.

[11] A. H. Sayed, Fundamentals of Adaptive Filtering. New York: Wiley, 2003.

[12] W. M. Younis, N. Al-Dhahir, and A. H. Sayed, "Adaptive frequencydomain equalization of space-time block-coded transmissions," in Proc. ICASSP, Orlando, FL, May 2002, pp. 2353-2356.

[13] W. M. Younis, A. H. Sayed, and N. Al-Dhahir, "Joint frequency-domain adaptive equalization and interference cancellation for multi-user space-time block-coded systems," in Proc. ICASSP, Hong Kong, Apr. 2003.

[14] $\longrightarrow$, "Adaptive frequency-domain joint equalization and interference cancellation for multi-user space-time block-coded systems," in Proc. Int. Contr. Conf., Anchorage, AK, May 2003.

[15] T. Kailath, Linear Systems. Englewood Cliffs, NJ: Prentice-Hall, 1980.

[16] W. M. Younis and A. H. Sayed, "Further complexity reduction of space-time block-coded receivers,", submitted for publication.

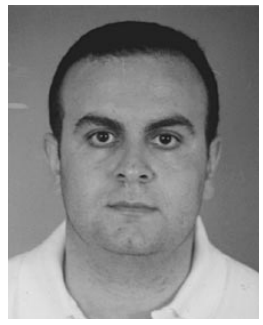

Waleed M. Younis (S'96) was born in Cairo, Egypt, in 1973. He received the B.S. and M.S. degrees in electrical engineering from Ain Shams University, Cairo, in 1995 and 1998, respectively. Since September 1999, he has been pursuing the Ph.D. degree in electrical engineering at the University of California, Los Angeles.

His research interests include adaptive filtering, equalization for wireless communications, spacetime coding, and MIMO communication systems.

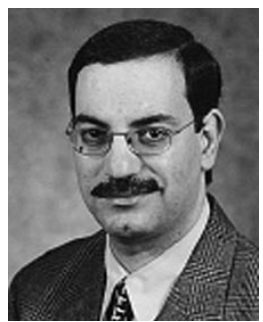

Ali. H. Sayed (F'01) received the Ph.D. degree in electrical engineering in 1992 from Stanford University, Stanford, CA.

$\mathrm{He}$ is currently Professor and Vice-Chairman of Electrical Engineering at the University of California, Los Angeles (UCLA). He is also the Principal Investigator of the UCLA Adaptive Systems Laboratory (www.ee.ucla.edu/asl). He has over 190 journal and conference publications, is the author of the textbook Fundamentals of Adaptive Filtering (New York: Wiley, 2003), and is coauthor of the research monograph Indefinite Quadratic Estimation and Control (Philadelphia, PA: SIAM, 1999) and of the graduate-level textbook Linear Estimation (Englewood Cliffs, NJ: Prentice-Hall, 2000). He is also co-editor of the volume Fast Reliable Algorithms for Matrices with Structure (Philadelphia, PA: SIAM, 1999). He is a member of the editorial boards of the SIAM Journal on Matrix Analysis and Its Applications and of the International Journal of Adaptive Control and Signal Processing. He has served as coeditor of special issues of the journal Linear Algebra and Its Applications. He has contributed several articles to engineering and mathematical encyclopedias and handbooks and has served on the program committees of several international meetings. He has also consulted with industry in the areas of adaptive filtering, adaptive equalization, and echo cancellation. His research interests span several areas including adaptive and statistical signal processing, filtering and estimation theories, signal processing for communications, interplays between signal processing and control methodologies, system theory, and fast algorithms for large-scale problems.

Dr. Sayed received the 1996 IEEE Donald G. Fink Award, a 2002 Best Paper Award from the IEEE Signal Processing Society in the area of Signal Processing Theory and Methods, and co-author of two Best Student Paper awards at international meetings. He is also a member of the technical committees on Signal Processing Theory and Methods (SPTM) and on Signal Processing for Communications (SPCOM), both of the IEEE Signal Processing Society. He is a member of the editorial board of the IEEE SIGNAL PROCESSING MAGAZINE. He has served twice as Associate Editor of the IEEE TRANSACTIONS ON SIGNAL PROCESSING, of which he is now serving as Editor-in-Chief.

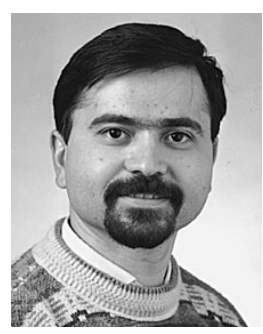

Naofal Al-Dhahir (SM'99) received the M.S. and Ph.D. degrees from Stanford University, Stanford, CA, in 1990 and 1994, respectively, both in electrical engineering.

He was an Instructor at Stanford University during the Winter of 1993. From August 1994 to July 1999, he was at the General Electric R and D Center, Schenectady, NY, where he worked on various aspects of satellite communication systems design and anti-jam GPS receivers. From August 1999 to July 2003, he was a principal member of technical staff at AT\&T Shannon Laboratory, Florham Park, NJ. Since August 2003, he has been an Associate Professor with the University of Texas at Dallas, Richardson. His current research interests include equalization schemes, space-time coding and signal processing, OFDM, wireless networks, and digital subscriber line technology. He has authored over 45 journal papers and holds ten U.S. patents in the areas of satellite communications, digital television, and space-time processing.

Dr. Al-Dhahir is a a member of the IEEE SP4COM technical committee. He is Editor for IEEE Transaction on Signal Processing, IEEE COMMUNICATIONS LETTERS, and IEEE TRANSACTIONS ON COMMUNICATIONS and will serve as co-chair of the Communication Theory Symposium at Globecom'04. He is co-author of the book Doppler Applications for LEO Satellite Systems (Boston, MA: Kluwer, 2001). 